\title{
An Evaluation of Image Based Steganography Methods
}

\author{
Kevin Curran, Internet Technologies Research Group, University of Ulster \\ Karen Bailey, Institute of Technology, Letterkenny, Ireland
}

Abstract - Steganography is a process that involves hiding a message in an appropriate carrier for example an image or an audio file. The carrier can then be sent to a receiver without anyone else knowing that it contains a hidden message. This is a process, which can be used for example by civil rights organisations in repressive states to communicate their message to the outside world without their own government being aware of it. Less virtuously it can be used by terrorists to communicate with one another without anyone else's knowledge. In both cases the objective is not to make it difficult to read the message as cryptography does, it is to hide the existence of the message in the first place possibly to protect the courier.

The initial aim of this study was to investigate steganography and how it is implemented. Based on this work a number of common methods of steganography could then be implemented and evaluated. The strengths and weaknesses of the chosen methods can then be analysed. To provide a common frame of reference all of the steganography methods implemented and analysed used GIF images. Seven steganography methods were implemented. The methods were chosen for their different strengths in terms of resistance to different types of steganalysis or their ability to maximise the size of the message they could store. All of the methods used were based on the manipulation of the least significant bits of pixel values or the rearrangement of colours to create least significant bit or parity bit patterns, which correspond to the message being hidden.

\section{INTRODUCTION}

The word steganography means "covered or hidden writing" [Johnson01]. The object of steganography is to send a message through some innocuous carrier (to a receiver while preventing anyone else from knowing that a message is being sent at all. Computer based stenography allows changes to be made to what are known as digital carriers such as images or sounds. The changes represent the hidden message, but result if successful in no discernible change to the carrier. The information may be nothing to do with the carrier sound or image or it might be information about the carrier such as the author or a digital watermark or fingerprint [Johnson01].

Cryptography and steganography are different. Cryptographic techniques can be used to scramble a message so that if it is discovered it cannot be read. If a cryptographic message is 
discovered it is generally known to be a piece of hidden information (anyone intercepting it will be suspicious) but it is scrambled so that it is difficult or impossible to understand and decode. Steganography hides the very existence of a message so that if successful it generally attracts no suspicion at all. Using steganography, information can be hidden in carriers such as images, audio files, text files, videos and data transmissions [Johnson01]. When the message is hidden in the carrier a stego-carrier is formed for example a stego-image. Hopefully it will be perceived to be as close as possible to the original carrier or cover image by the human senses. Images are the most widespread carrier medium [Westfield99]. They are used for steganography in the following way. The message may firstly be encrypted. The sender (or embedder [Pfitzmann96]) embeds the secret message to be sent into a graphic file [Zollner98] (the cover image [Pfitzmann96] or the carrier). This results in the production of what is called a stego-image. Additional secret data may be needed in the hiding process e.g. a stegokey. The stego-image is then transmitted to the recipient [Zollner98]. The recipient (or extractor [Pfitzmann96]) extracts the message from the carrier image. The message can only be extracted if there is a shared secret between the sender and the recipient. This could be the algorithm for extraction or a special parameter such as a key [Zollner98] (the stegokey). A stegoanalyst or attacker may try to intercept the stego-image. Figure 1 below shows the steganographic system.

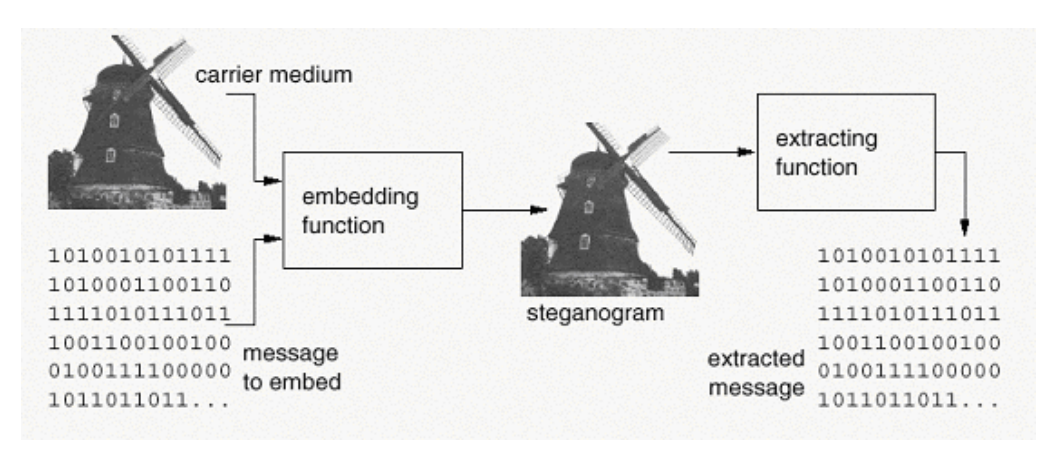

Figure 1 : The Steganographic System [Westfield99].

To make a steganographic communication even more secure the message can be compressed and encrypted before being hidden in the carrier. Cryptography and steganography can be used together. If compressed the message will take up far less space in the carrier and will minimise the information to be sent. The random looking message which would result from encryption and compression would also be easier to hide than a message with a high degree of regularity. Therefore encryption and compression are recommended in conjunction with steganography [Fridrich99]. 


\subsection{TYPES OF DIGITAL CARRIERS}

There are a variety of digital carriers or places where data can be hidden. Data may be embedded in files at imperceptible levels as noise. Properties of images can be manipulated including luminescence, contrast and colours [Johnson01]. In audio files small echoes or slight delays can be included or subtle signals can be masked with sounds of higher amplitude. Information can be hidden in documents by manipulating the positions of the lines or the words. When HTML files are written web browsers ignore spaces, tabs, certain characters and extra line breaks. These could be used as locations in which to hide information. Messages can be retrieved from text by taking for example the second letter of each word and using them to produce the hidden message. This is called a null cipher or open code [Johnson01]. Information can be hidden in the layout of a document for example certain words in a piece of text can be shifted very slightly from their positions and these shifted words can then make up the hidden message. The way a language is spoken can be used to encode a message such as pauses, enunciations and throat clearing [Johnson01].

Unused or reserved space on a disc can be used to hide information. The way operating systems store files typically results in unused space that appears to be allocated to the files. A minimum amount of space may be allocated to files but the file does not need all this space so some of it goes unused. This space can be used to hide information. Another method for hiding information in file systems is to create a hidden partition [Johnson01]. Data may be hidden in unused space in file headers. Packets for example TCP / IP packets have headers with unused space and other features that can be manipulated to embed information [Johnson01]. Data can be hidden using the physical arrangement of a carrier for example the layout of code in a program or electronic circuits on a board. This process can be used to record and identify the origin of the design and cannot be removed without a substantial change to the physical layout. Spread spectrum techniques can also be used by placing an audio signal over a number of different frequencies. Random number generators are developed to allow spread spectrum radios to hop from frequency to frequency. Systems can use different frequencies at the same time. Some information is broadcast on one frequency and some on another. The message can be reassembled by combining all the information [Wayner02]. 


\subsection{IMAGE STRUCTURE AND IMAGE PROCESSING}

A digital image is the most common type of carrier used for steganography. A digital image is produced using a camera, scanner or other device. The digital representation is an approximation of the original image [Efford00]. The system used for producing the image focuses a two dimensional pattern of varying light intensity and colour onto a sensor [Efford00]. The pattern has a co-ordinate system and the origin is the upper left hand corner of the image. The pattern can be described by a function $f(x, y)$. An image can be described as an array of numbers that represent light intensities at various points. These light intensities or instances of colour are called pixels. Sampling is the process of measuring the value of the image function $\mathrm{f}(\mathrm{x}, \mathrm{y})$ at discrete intervals in space [Efford00]. Each sample is the small square area of the image known as the pixel. The raster data of an image is that part of the image that can be seen i.e. the pixels [Johnson01]. The size of an image can be given in pixels, for example an image which is $640 \mathrm{x} 480$ pixels contains 307,200 pixels [Johnson01]Pixels are indexed by $\mathrm{x}$ and $\mathrm{y}$ co-ordinates with $\mathrm{x}$ and $\mathrm{y}$ having integer values [Efford00]. The spatial resolution of an image is the physical size of the pixel in the image. Dense sampling produces a high-resolution image in which there are many pixels and each contributes a small part of the scene. Coarse sampling results in a low-resolution image in which there are fewer pixels [Efford00]. The rate of change of the value $f(x, y)$ as it moves across the image is the spatial frequency. Gradual changes in $\mathrm{f}(\mathrm{x}, \mathrm{y})$ correspond to low spatial frequencies and can be represented in a coarsely sampled image. Rapid changes correspond to high spatial frequencies and must be represented by a densely sampled image. The Nyquist criterion states that the sampling frequency should be at least double the highest spatial frequency found in the image. A coarsely sampled image that does not follow this criterion may suffer from the effects of aliasing [Efford00] shown in Figure 2 below.

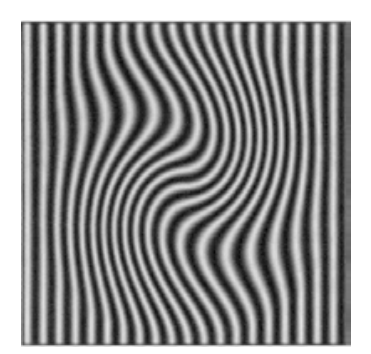

a.

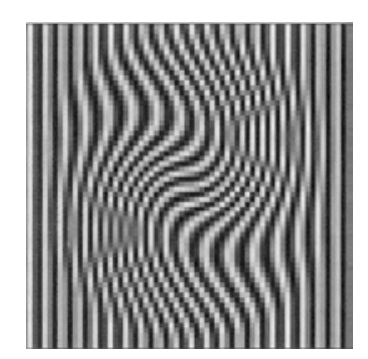

b.

Figure 2: a. Image without Aliasing b. Coarsely Sampled Image Showing Aliasing Artefacts (Efford, 2000). 
Each pixel is generally stored as 24-bit or 8-bit. A 24-bit pixel has a possibility of $2^{24}$ colour combinations [Johnson01]The 24 bits of a 24-bit image are spread over three bytes and each byte represents red, green and blue respectively. Colours are obtained by mixing red, green and blue light in different proportions. An image can be formed by making three measurements of brightness at each pixel using the red, green and blue components of the detected light. Using the RGB model the value of $f(x, y)$ is a vector with three components corresponding to red (R), green $(\mathrm{G})$ and blue (B). They can be regarded as orthogonal axes defining a three dimensional colour space. Every value of $f(x, y)$ is a point in the colour cube shown in Figure 3 below. The three components are normally quantised using 8 bits. An image made of these components is described as a 24-bit colour image [Efford00].

Each byte can have a value from 0 to 255 representing the intensity of the colour. The darkest colour value is 0 and the brightest is 255 . For example a pixel could be made up of three bytes as follows: 111111110000000000000000 . The first 8 bits represent red, the second 8 bits represent green and the third 8 bits represent blue. The bit values in this example result in a red pixel. Its red byte is at a maximum value (11111111) and its green (00000000) and blue (0000000) bytes have the lowest possible value. Transparency is controlled by the addition of information to each element of the pixel data. This is to allow image overlay [Murray96]. A 24-bit pixel value can be stored in 32 bits. The extra 8 bits specify transparency. This is sometimes called the alpha channel [Murray96]. An ideal 8-bit alpha channel can support transparency levels from 0 (completely transparent) to 255 (completely opaque). It can be stored as part of the pixel data [Murray96] e.g. RGBA (red, green, blue and alpha taking up 4 bytes in total).

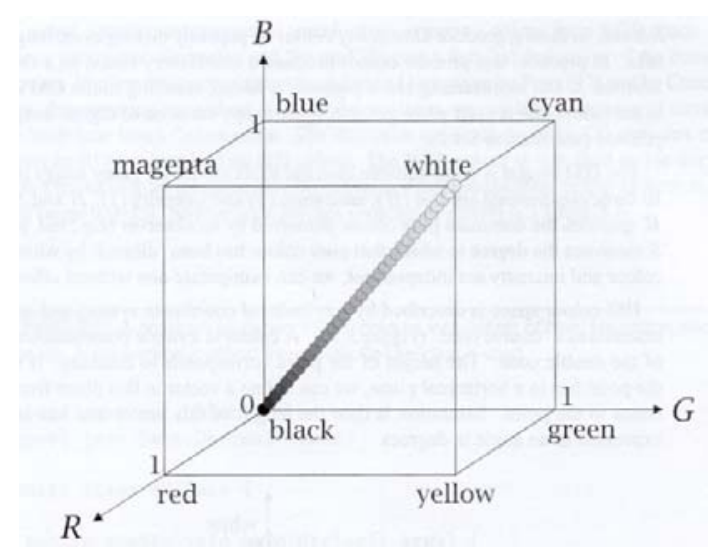

Figure 3 : The RGB Colour Cube (Efford, 2000)

Some images are 8-bit. Each pixel is represented by one byte only. This one byte can have any value ranging from 0 to 255,256 possible colours or 256 greyscale values for black and www.ijde.org 
white images. The colours are taken from a colour index or palette also called a colour map or colour table. This palette contains up to 256 colours representing the colours in the image. The value of the pixel in an image points to a colour in the palette [Johnson01]The GIF (Graphic Interchange Format) image format uses this process. When a GIF image is displayed the software paints the specified colour from the palette onto the screen [Johnson01] at each pixel. If the image has fewer colours than the size of the palette any unused colours in the palette are set to zero [Murray96] GIF is a bitmap image. Bitmap is a system in which an image is described as a bit pattern or series of numbers that gives the shade or colour of each pixel [Day98]. In true greyscale images, values from 0 to 255 represent the intensity of the colour and do not refer to a palette [Johnson01]A palette image format contains a header, a palette and image data (pointers to the palette).

There are two steps in creating palette-based images - colour quantisation and dithering [Fridrich99]. A colour quantisation algorithm has two parts, generating the colour palette and mapping the pixels. To generate the palette, colours are extracted from the image. Each pixel in the image is then mapped to its nearest colour in the palette to generate the quantized image [Hsieh99]). Quantisation involves replacing a continuously varying $\mathrm{f}(\mathrm{x}, \mathrm{y})$ with a set of quantisation levels [Efford00]. A set of quantisation levels comprises the integers $0,1,2,3 \ldots$ $\mathrm{n}-1.0$ and $\mathrm{n}-1$ are displayed as black and white with intermediate levels in shades of grey (greyscale). The number of grey levels is usually an integral power of $2 . n=2^{b}$ where $b$ is the number of bits used for quantisation. It is typically 8 resulting in images with 256 grey levels ranging from black to white [Efford00]. There are different algorithms for quantisation. Colour quantisation involves truncating all the colours of the original 24-bit image to a finite number of colours, 256 for GIF, 216 for Netscape GIF and 2 for black and white. Splitting algorithms split the colour space of the original image into two subspaces according to some preference criteria (Hsieh and Fan, 1999). The splitting is iteratively carried out until the correct number of subspaces is reached. The colour representing the subspace becomes the quantized colour. Clustering methods can also be used in which colours are clustered to form the quantized colours. The method usually used for quantization involves iterative dividing of a three dimensional colour cube into two boxes with approximately the same number of colours. The half with the largest dimensions is chosen by measuring either the greatest difference in RGB value or the greatest difference in luminosity [Wayner02]. The half with the largest dimensions is selected and the iteration is continued until the desired number of colours is produced [Fridrich99]. The centres of gravity of each box are then rounded to integer colours representing the colours of the palette. The largest dimension can be replaced using the largest standard deviation resulting in a slightly better algorithm [Fridrich99]. It 
should be noted that standard quantisation algorithms will not necessarily yield exactly 256 colours in the image.

Dithering is a technique used to simulate colours that are missing from an images palette. This is done by intermingling pixels of two or more palette colours. Colours are reordered so that their visual combination matches the original images more closely. If the unavailable colour differs too much from the colours in the palette a grainy appearance results [Johnson01] and errors are present in the form of false contours. Dithering can be based on error diffusion. The image is scanned in some regular way for example by rows. The colour of a pixel is rounded to its closest colour in the palette. This produces an error. The error is negative if the rounding has resulted in a decrease in the pixel value. The error is positive if the rounding has increased the pixel value. The error is multiplied by weights and added to the surrounding pixels that have not yet been visited. Using this method the rounding error is spread to neighbouring pixels which results in an image that is more visually pleasing [Fridrich99]. This process is repeated for every colour in the image [Johnson01]. 


\section{STEGANALYSIS}

There are two stages involved in breaking a steganographic system: detecting that steganography has been used and reading the embedded message [Zollner98]. Steganalysis methods should be used by the steganographer in order to determine whether a message is secure and consequently whether a steganographic process has been successful. The goal of a stegoanalyst is to detect stego-messages, read the embedded message and prove that the message has been embedded to third parties [Pfitzmann96]. Detection involves observing relationships between combinations of cover, message, stego-media and steganography tools [Johnson01]. This can be achieved by passive observation. Active interference by the stegoanalyst involves removing the message without changing the stego-image too much (the stegoanalyst might want to conceal his existence), or removing the message without consideration to the stego-image appearance or structure [Pfitzmann96]. Whether a message has been hidden in an image or not the image could be manipulated to destroy a possible hidden message [Johnson98].

There are two necessary conditions to be fulfilled for a secure steganographic process. The key must remain unknown to the attacker and the attacker should not be familiar with the cover image [Zollner98]. If the cover image is known, the message could then be embedded in a random way so that it is secure however it is preferable that the image is unknown. Attacks on steganography can involve detection and/or destruction of the embedded message. A stego-only attack is when only the stego-image is available to be analysed [Johnson98]. A known cover attack is when the original cover image is also available. It involves comparing the original cover image with the stego-image. As explained above hiding information results in alterations to the properties of a carrier which may result in some sort of degradation to the carrier [Johnson98]. Original images and stego-images can be analysed by looking at colour composition, luminance and pixel relationships and unusual characteristics can be detected. If a hidden message is revealed at some later date the attacker could analyse the stego-image for future attacks. This is called known message attack. The chosen stego attack is used when the steganography algorithm and the image are known. A chosen message attack is when the stegoanalyst generates stego-images using a given steganography algorithm using a known message [Johnson98]. The purpose is to examine the patterns produced in the stego-images that may point to the use of certain steganography algorithms. Most steganographic algorithms embed messages by replacing carefully selected pixels bits with message bits [Westfield99]. Any changes to the data associated with the image through embedding will change the properties of the image in some way. This process may create patterns or unusual exaggerated noise [Johnson98]. An image with plenty of bad effects is a problem that can be 
detected with the human eye. The patterns visible to the human eye could broadcast the existence of a message and point to signatures of certain methods or tools used [Johnson98]. Human sight is trained to recognise known things. This process of analysis depends on the ability of humans to discern between normal noise and visual corruption and patterns created by steganography [Westfield99]. It can be difficult to distinguish randomness and image contents and to distinguish LSBs and random bits by machine.

Visual attacks can involve removing the parts of the image containing the message i.e. the least significant bit of each pixel [Westfield99]. Sometimes the least significant bits are not very random. The human eye may then distinguish whether there is a message distorting the image content. The filtering process which can be used for this purpose involves extraction of the potential message bits based on a presumed steganographic method and then illustrating the bits on the position of their source pixels [Westfield99].

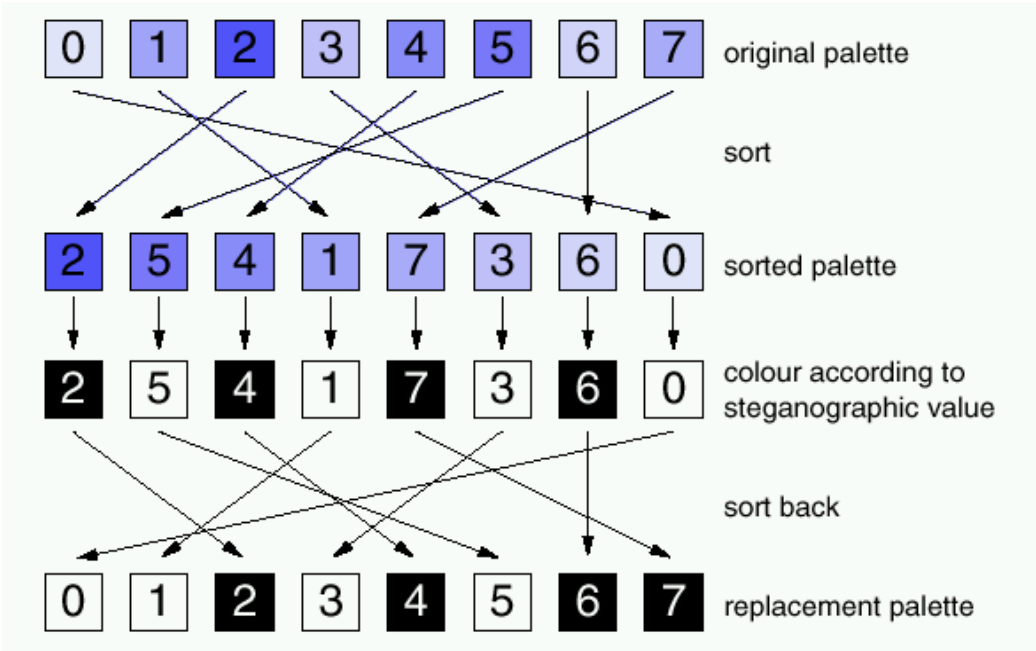

Figure 4 : Assignment function of replacement colours; colours that have an even index in the sorted palette become black, the rest becomes white [Westfield99]

An embedding filter presents the values pixels yield when the extraction function is applied to them. For example the embedding filter for EzStego replaces the original palette by a black and white palette. The original palette is sorted by frequency. The palette is then sorted by luminance. Every even position in the palette $(0,2,4,6, \ldots)$ is set to black and every uneven position in the palette is set to white. [Westfield99]. The replacement palette is now reordered into its original order. This is shown in Figure 4 above.

If numerous comparisons can be made between the cover images and the stego-images patterns can begin to emerge [Johnson01]. At a later stage if the cover is not available the $\underline{w w w . i j d e . o r g}$ 
known signature will be sufficient to indicate a message and the tool used to embed it [Johnson01]. Some of the methods of carrying out steganography produce characteristics that act as signatures for that steganography method [Johnson98]. The image may not give away the existence of stenography but the palette could. Therefore steganography can be detected by examining the palette itself. In colour palettes the colours are ordered from most used to least used. The changes between colour values rarely change in one-bit increments in an unstegoed image. But this feature would be created by embedding in the LSBs during steganography. Greyscale palettes do change in one bit increments but all the RGB values are the same. In monochromatic images usually two of the RGB values are the same and the third usually has a much stronger saturation of colour. Therefore there are expected patterns in palettes which if adjusted could indicate the use of steganography. If an original image contains 200 colours steganography could result in the formation of 400 colours which would be too many to store in the palette. When the image is saved as an 8-bit image it will produce a new palette with 256 colours and information hidden could be lost. This is prevented by reducing the colours initially so that space is available for new colours to be created. Adjacent colours are added which are very close to the original. A stego-image is produced which is very close to the original cover image [Brown94]. Reducing the colours in the palette and creating new colours resulting from changing the LSBs of existing colours will results in blocks of similar colours differing by one bit in the palette. This is shown in figure 2.14 . The palette on the right has blocks of similar colours produced by the creation of new colours by adjustment of the LSBs. A unique pattern has been created in the palette.
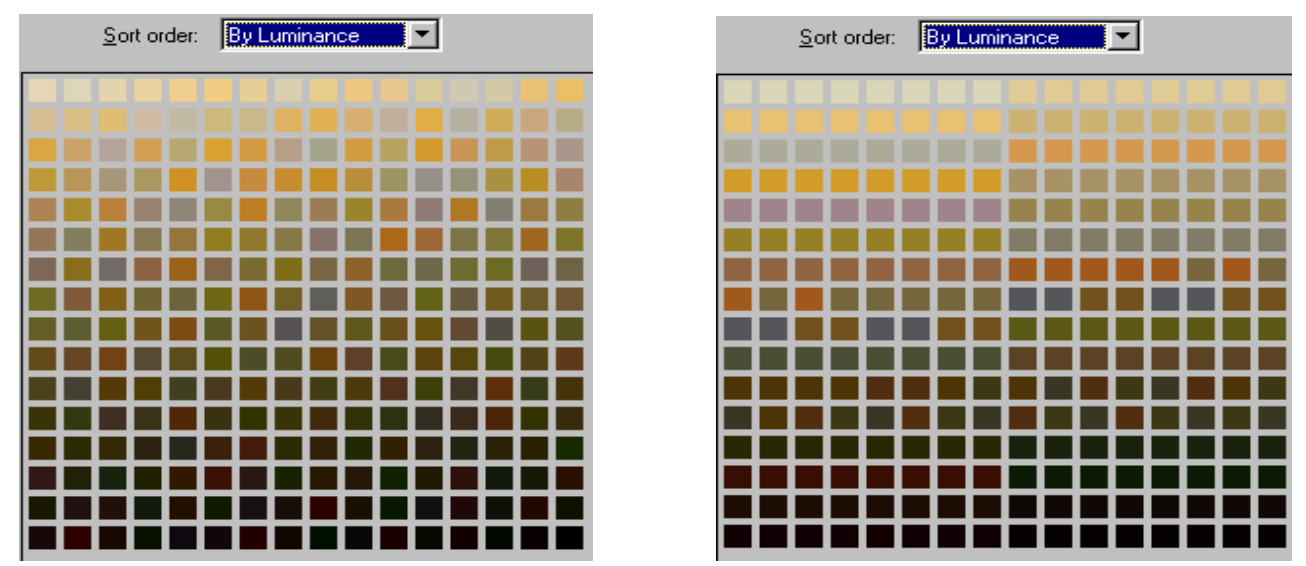

Figure 5 : Cover (left) and Stego-Image Palette (right) after S-Tools [Johnson98]

Using a steganography method where pointers to the palette are changed may increase noise because adjacent colours in the image become very different after the message has been embedded [Johnson98]. This is alleviated by the use of greyscale images [Johnson98]. A 
method of detection might be to look at areas in the image where colour does not flow well from one area to another. If pixels beside each other have very different colours this could indicate the existence of steganography [Wayner02]. If images are created which have very different adjacent palette entries, small shifts in the LSBs of the pixel colours will cause radical colour changes in the image advertising the existence of a hidden message. The palette may not be altered but changes to the pixel colours may show dramatic changes to the image [Johnson98]. Steganography tools that modify the lower bits of 8-bit images can produce noisy distorted stego-images [Hansmann96]. In Figure 6 below the left image is the original and the right image shows the impact of embedding using Hide and Seek which is a steganographic method that results in changes to the LSBs.
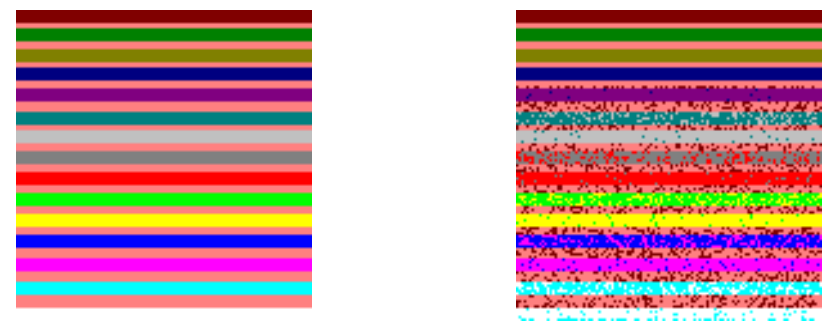

Figure 6: Original 8-bit Cover Image (left), and the 8-bit Stego-Image (right)

\section{Created with Hide and Seek. [Johnson98]}

An 8-bit image could be converted to a 24-bit image to produce a less noisy or distorted stego-image. Colour values can be directly manipulated and any changes will most likely be visually undetectable. However the image could end up quite big and unsuitable for electronic transmission [Johnson98]. Detection of a hidden message may be related to the size of the image and the format and content of the carrier image. If the message is larger an image will receive more modification. There is then a higher probability that the modifications will be detected [Fridrich99] and consequently there is then an increased probability the message will be detected [Fridrich00]. Statistical attacks can be carried out using automated methods. A stego-image should have the same statistical characteristics as the carrier so that the use of a stenographic algorithm can't be detected [Westfield99]. Therefore a potential message can be read from both the stego-image and the carrier and the message should not be statistically different from a potential message read from a carrier [Westfield99]. If it were statistically different the steganographic system would be insecure. Automation can be used to investigate pixel neighbourhoods and determining if an outstanding pixel is common to the image, follows some sort of pattern or resembles noise. A knowledge base of predictable patterns can be compiled and this can assist in automating the detection process [Johnson98]. Steganalysis tools can determine the existence of hidden messages and even the tools used to 
embed them. A person wishing to detect someone else's stegoed images may analyse the type of equipment being used to create the image such as a scanner or digital camera [Fridrich99]. A set of statistical measures could be determined which satisfy all of the images produced by this piece of equipment and consequently statistical fingerprints could be determined caused by the presence of a hidden message [Fridrich00].

In order to prevent detection steganographic and cryptographic keys can be used. A steganographic key controls embedding and extracting of the message. The key could scatter the message randomly over the carrier. A cryptographic key is used to encrypt a message before embedding. Therefore even when the message is detected it can't be read. In the case of bitwise methods destruction of the embedded message is fairly easy because the LSBs of the images can be changed with compression. The image may be converted to lossy compression format such as JPEG. JPEG images which have been processed with Jpeg-Jsteg can be recompressed and this will destroy the message embedded in the DCT coefficients because they will be recalculated [Johnson98].

A series of tests were carried out by Johnson to distort embedded data. The purpose was to evaluate the robustness of bit-wise and transform tools [Johnson98]. The object of the tests was to show what the techniques will withstand and what are some common vulnerabilities. Images tested included digital photographs (24-bit or 8-bit greyscale JPEG and 24-bit BMP), clip art (8-bit GIF) and digital art (24-bit BMP or JPEG, 8-bit BMP or GIF). To determine robustness images were manipulated following embedding with a number of image processing techniques: converting between lossless and lossy formats, converting between bit densities, blurring, smoothing, adding noise, removing noise, sharpening, edge enhancement, masking, rotating, scaling, re-sampling, warping, converting from digital to analog and back (printing and scanning), mirroring, flipping, adding bitwise messages, adding transform messages and applying the unZign and StirMark tools to test the robustness of watermarking software. Tests were also carried out to determine the smallest size of image that can be used. The tests were used to alter the information to such a point that it could not be retrieved again. The message was then checked.

Conversion to compressed JPEG images or minor processing disables the bit wise tools. Tools that relied on bit wise methods to hide the data could not recover any of the messages. The transform tools did survive several of the image processing tests but failed when combinations of image processes were carried out on them. These tests showed that the transform methods are in fact more robust. 


\section{IMAGE ANALYSIS}

Having carried out a review of steganographic and cryptographic methods it has been found that one of the main methods typically used for steganography involves the process of hiding a message in image pixels. Images are the most widespread carrier medium used. There are various ways of doing this. In some methods the purpose is to minimize changes to the image and in some the purpose is to store the message in a random way so as to make it more difficult to detect. In some methods more information can be stored by using more than one bit of the colours representing the pixels. This allows more information to be stored but also results in the formation of more new colours and the need to use an image that is comprised of less colours to start with. In some methods the process of storing information can result in the production of new colours in the image and in some methods the existing colours only are used. Some methods involve the use of a key. These different characteristics may be used individually or combined to produce similar systems to many of those currently being used. The aim of this study is to produce a system containing different steganographic methods and to examine the strengths and weaknesses of those methods. The system to be produced will also contain an option to encrypt the message before it is embedded.

While steganography can be carried out on any digital media it is reasonable for the purposes of cross comparing methods to implement these methods on a common media type. The chosen media for this system are GIF images. GIF images have been chosen because as they are widely used in web pages, are recognised by all browsers and they are easily distributable which ensures that they lend themselves to this type of activity without drawing attention to themselves. Audio and video carriers require more intensive processing to hide data in them as the files the carrier is stored in tend to be larger. BMP files have a very large number of colours, which has advantages from a steganography point of view, but their corresponding size makes them unsuitable for distribution across a web type medium. It has also been found that the compression / decompression algorithm causes problems for steganography. GIF undergoes lossless compression. The advantage of lossless compression is that the original digital image is reproduced exactly and therefore the original arrangement of bits making up the image is maintained. The LZW technique must normally be paid for however the software is included in Java so that working on GIF programs in Java does not require any payment. Images saved in JPEG use lossy compression in which the image is not reconstructed in exactly the same way as the original. Space is saved in storing the image however some of the bits and hence some of the data can be lost. JPEG files are sensitive to small changes in the image data, which results in less capacity for holding a message ${ }^{9}$. It raises less suspicion than 
for example a BMP format ${ }^{11}$. Many steganograhy packages use GIF images including Hide and Seek, S-Tools, GifShuffle, EzStego, and the method developed by Fridrich. A GIF image has a restricted number of colours. The maximum number it can have is 256 . GIF is a bitmap image. Bitmap is a system in which an image is described as a bit pattern or series of numbers that gives the shade or colour of each pixel. Greyscale GIF images, have pixel values from 0 to 255 which represent the intensity of the colour and do not refer to a palette ${ }^{2}$. Therefore the purpose of this study is to develop a package which will carry out steganography by hiding a text message in a GIF image carrier. As many of the methods which carry out steganography use the process of hiding the message in image pixels this system will focus on hiding the message in the least significant bits or parity bits of the colours in the pixels of an image. It is proposed to develop and evaluate the following methods explained below. The names of the methods are used to distinguish between the different steganographic processes that will be carried out.

\subsection{STEGO ONE BIT}

When images are used as the carrier in steganography they are generally manipulated by changing one or more of the bits of the byte or bytes that make up the pixels of an image. The message can be stored in the LSB of one colour of the RGB value or in the parity bit of the entire RGB value. Hide and Seek uses GIF images and the lower order bit of each pixel. One of the methods for S-Tools involves changing the least significant bit of each of the three colours in a pixel in a 24-bit image. Changing the LSB will only change the integer value of the byte by one. This will not noticeable alter the visual appearance of a colour and hence the image itself. Changing a more significant bit would cause a proportionately greater change in the visual appearance of a colour. The main objective of steganography is to pass a message to a receiver without an intruder even knowing that a message is being passed which means that there should be no discernable change to the carrier. This is the first method to be tested and will involve encoding some of the basic processes required for later steganographic methods to be tested also. It will involve changing the LSB of one of the colours making up the RGB value of the pixel. This should have very little effect on the appearance of the image. This process will most likely result in the formation of new colours for the palette. Therefore the image used must have a palette size of 128 pixels or less. This will allow for a doubling of the colours in the palette (the creation of a new colour for every existing colour in the palette) which is the maximum number of colours that could be produced by this method. It may be found that if the palette is ordered by luminance that there will be pairs of very similar colours. How noticeable that is depends on the colour profile used in the image to start with. Practical methods should allow for the use of the full image size, thus the amount of data that 
can be hidden is proportionate to the number of pixels in the image rather than to the colours in the palette. The only restriction is then the size of the image. Using the image data for embedding is less restrictive on capacity compared to another method where data is stored in the palette itself. Using a 128 palette image should not result in too much distortion to the original image.

\subsection{STEGO TWO BITS}

Using this method two LSBs of one of the colours in the RGB value of the pixels will be used to store message bits in the image. This will involve using a palette with a maximum of 64 colours allowing for the production of a possible 192 new colours,i.e. three new colours for each existing colour. Less colour will be available to represent the starting image and hence it will be more degraded than the image used in the method Stego One Bit. The advantage of this method is that twice as much information can be stored here than in the previous method. This method could instead have used the LSB of two colours in the RGB value which would have resulted in the same amount of storage space. The starting image would still have to have a palette containing 68 colours.

\subsection{STEGO THREE BITS}

Using this method three LSBs of one of the colours in the RGB value of the pixels will be used to store message bits. This will involve using a palette with a maximum of only 32 colours allowing for the production of a possible 224 new colours, three new colours for every existing colour in the image. The data hiding capacity is 3 times the storage capacity of Stego One Bit but the image will be even more distorted than if a 128 colour palette was used.

\subsection{STEGO FOUR BITS}

Using this method four LSBs of one of the colours in the RGB value of the pixels will be used to store message bits. This will involve using a palette with a maximum of only 16 colours allowing for the production of a possible 240 new colours. This is the smallest palette that could be used for an image using Jasc Paint Shop Pro. The colours are now very restricted but an area of one particular colour in the image may have 16 variations distributed through it which could result in a certain amount of texture mitigating the effects of such a restricted palette.

\subsection{STEGO COLOUR CYCLE}

In order to make the detection of the hidden data more difficult it was decided to cycle through the colour values in each of the pixels in which to store the data. This also means that the same colour was not constantly being changed. For example the first data bit could be www.ijde.org 
stored in the LSB of the blue value of the pixel, the second data bit in the red value and the third data bit in the green value, The alpha value will be skipped and the next colour used will be blue again. This is because changing the alpha value which is generally 255 would look too suspicious unless the image used contained different transparency levels.

\subsection{STEGO1BITPRNG}

A pseudo random number generator can be used to choose random pixels in which to embed the message. This will make the message bits more difficult to find and hopefully reduce the existence of patterns in the image. Most importantly it means that if a cracker removed the LSBs from one of the colours and tried to read them it would make no sense as they would not be in order. A pseudo random number generator will be created and will be used to select the pixels in which to hide the data. Data will then be hidden in the LSB of the blue value. If the message is much smaller than the capacity of the image a problem may occur whereby the information will be packed into one part of the image for example the top half. This is solved by using a PRNG which will spread the message all over the image. Hide and Seek arranges it so that the message bits will not be beside one another but instead randomly dispersed throughout the image. Hence the noise will also be randomly distributed. A user chosen key can be inserted into a pseudo random number generator which will determine a sequence of random numbers. These numbers will indicate the pixels in the image where the least significant bit is to be changed. This makes the system more secure because the reader of the message must know the key in order to determine in which bytes the message bits are hidden. The key must remain unknown to the attacker. If the cover image was known to the attacker, embedding the message in a random way would improve its security.

\subsection{STEGOFRIDRICH}

EzStego encodes in the parity bit of indices of a GIF image. Fridrichs newer method also involves manipulating existing colours in the palette. EzStego however firstly orders the palette by luminance so that similar colours are beside one another. But Fridrichs method involves pairing up all the colours in the palette so that the distance between the two colours in each of the pairs is minimized. This method searches for the closest colour to the colour of the pixel which has the correct parity for the bit to be hidden. The message is hidden in the parity bit of the RGB values of close colours. For the colour of each pixel into which a message bit is to be embedded the closest colours in the palette are searched until a palette entry is found with the desired parity bit. This technique does not change the palette in any way either by ordering it or by increasing the colours present in it. The parity bits of palette entries of real images are randomly distributed therefore using this method it is never 
necessary to depart from the original colour too much. This avoids the problem of occasionally having to make large changes in colour which might indicate that a message has been hidden. Fridrich claims that his/her method of pseudo randomly changing the LSB of a pixel by locating the closest pixel colour in the palette rather than adjusting the palette as with EzStego produces approximately four times less distortion to the carrier image. Fridrich finds the distance between colours whereas EzStego orders the palette by luminance. This is the final steganography method to be encoded and evaluated. It is based on the method of Fridrich but instead of searching for the closest colour each time a bit is to be hidden in a pixel the closest colour to each colour in the palette with the opposite parity bit is initially chosen. This reduces the problem of having to search through the palette each time a bit is to be hidden. The pixels in which to hide the message are also pseudo randomly chosen in this study a technique which Fridrich also uses.

\subsection{CRYPTOGRAPHY - KNAPSACK}

An encryption method will be investigated and will be provided as one of the final options for the user. The purpose here is not to use or develop a secure cryptographic method but to use a relatively simple method which contains a key in order to more randomly distribute the message over the image. Knapsack was the first algorithm for generalized public key encryption. Unfortunately it was found to be insecure. The idea behind Knapsack is that, given a pile of items each having different weights it is possible to put some of them into a Knapsack so that the Knapsack weighs a given amount. Given a set of values $M_{1}, M_{2}, M_{3}$, $\ldots . . M_{n}$ and a sum $S$ the values of $b_{i}$ are computed so that $S=b_{1} M_{1}+b_{2} M_{2}+b_{3} M_{3}+$ $\ldots . b_{n} M_{n}$. The values of $b_{i}$ can be either 0 or 1.1 means that the value is in the sack 0 means that it is not. This method has a public key and a private key. The private key is a superincreasing sequence of numbers. A series of bits related to the plaintext are compared with the key. Values in the key which have a 1 opposite them are added to the sum S i.e. the total weight. A series of numbers generated in this manner builds up and this becomes the encrypted code. A public key is also used. All of the values in the super-increasing sequence are multiplied by a number $n$. mod $\mathrm{m}$. The modulus must be a number greater than the sum of all the values in the super-increasing sequence. $\mathrm{n}$ the multiplier should have no factors in common with $\mathrm{m}$. The sequence of numbers produced is the public key.

\section{STEGANOGRAPHY SYSTEM}

The best types of images to use are black and white greyscale or natural photographs with 24 bits per pixel which have been scanned in. The redundancy of the data helps to hide the 
presence of a secret message. A cover image should contain some randomness. It should contain some natural uncertainty or noise. Hiding information may introduce enough visible noise to raise suspicion. Therefore the carrier or cover image must be carefully selected. Once it has been used the image should not be used again and should be destroyed. A familiar image should not be used so it is better for the steganographer to create his / her own images. Some software displays the image before and after data is hidden. This will also be done here.

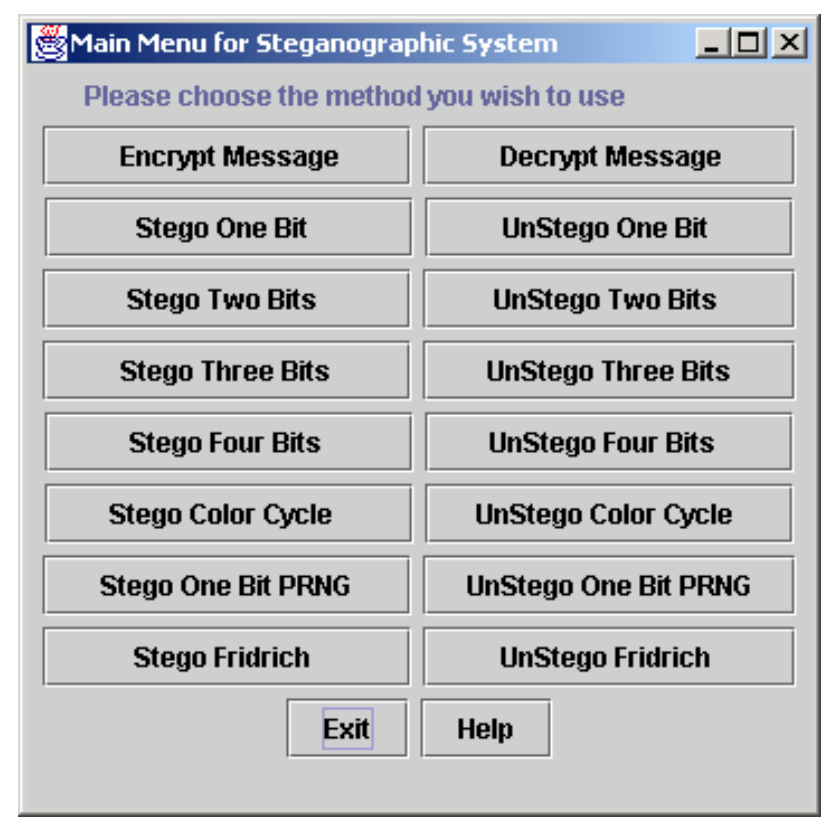

Figure 7: Steganography Software GUI

The user will be someone who is familiar with the process of information hiding and will have knowledge of Information systems. Cryptography is recommended and will result in a more random looking message rather than a high degree of regularity. A cryptographic method will be included as an option prior to steganography. The user should be able to select a plaintext message from a file, an image to be used as the carrier and then select a steganographic method, which will hide the selected message in the selected carrier image. The user will then be able to save the stegoed image in another file. The user should also be provided with the option to encrypt the message prior to hiding it in the image. The user should be able to open an image file containing a stegoed image containing a message to be read and choose an appropriate method to unstego the message from the image. The user should then be provided with the hidden message. A graphical user interface will be provided for the user to select the appropriate files and methods. The software provides a GUI, which will allow a user to select a file containing the message, the image in which to store the message and a file in which to store the stegoed image. The user will also be able to select the method for steganography and for encryption if desired. The opening frame of the graphical 
user interface which is shown in Figure 7 contains a series of buttons one, to encrypt a message, one to decrypt a message, one for each of the seven steganography methods and one for each of the seven methods to reverse the steganography process and finally a Help button and an Exit button.

\section{EVALUATION}

The next and possibly the most important stage is the evaluation of the steganographic methods implemented above. Based on the literature survey a number of evaluation methods have been chosen. The steganographic methods will be evaluated by passive observation of the stegoed image and by comparing the stegoed image with similar unstegoed images. Similar here means that they are not the same but have the same content and lighting. Evaluators will look for patterns or unusual exaggerated noise. The patterns visible to the human eye could broadcast the existence of a message. Another type of analysis involves comparing the original cover image with the stego-image in a number of ways. The palette will be examined. A reduced palette or noticeable pairs of colours in the palette will be looked for. A palette containing only 128 different colours should be easy to detect. The effects of embedding larger and smaller messages will be evaluated. Colour and greyscale images will be compared. Images were created for this study using a digital camera. The content for the images chosen was natural unsymmetrical environments with fairly busy subject matter. Smooth continuous areas are minimized and the images contain quite a high level of detail. In order to minimize the number of different variables which had to be taken in account in the evaluation process it has been decided not to encrypt the messages hidden in the stegoed images. Thus the Knapsack encryption algorithm has been implemented but is not included in the evaluation process.

\subsection{METHODS OF EVALUATION}

Four distinct but complementary methods of evaluation will be used. These are:

- Pattern Analysis of Image Pixels: This detection method is based on looking for patterns in the bits that make up the pixel colours. For example if methods hide messages in the least significant bits of pixels then looking for patterns in the least significant bits of pixels is an easy way to detect the existence of messages. There are many variations of this message hiding technique such as hiding the message bit in the least significant bit of either the red, green or blue value of the colour or the parity bit of the pixel. This analysis 
will be carried out based on the literature survey rather than visual inspection of the images. The objective is to determine which of the methods being studied are vulnerable to this method of analysis and how vulnerable they are.

- Pattern Analysis of Image Palette: This detection method is based on looking for patterns in the images palette. For example some steganography methods require an image with a reduced number of colours. The steganography methods then create new colours that are almost identical to the existing ones but have different least significant bits or parities. By ordering the palette by luminance such pairings may be visible. The fewer original colours the steganography method allows the more obvious it should be in the palette. A colour reduced original image with a large message may have up to 256 colours in the palette half of which will be almost identical to the other half. With a small message the number of new colours added to the original reduced set will be much smaller. In practice however the number of new colours created by the steganography process must be a maximum of 256 (GIF images) but is otherwise random. Some methods do not however use colour-reduced images and rely entirely on existing colours.

For this part of the analysis the palette of a greyscale image was compared 7 times, once for each different type of steganography method. Palettes will be examined for colour images which have had various forms of steganography carried out on them. In each case the palette will be ordered by luminance to make it more coherent. Of course when the palette is not ordered by luminance, patterns in the palette are much harder to see. It is assumed however that somebody attempting to carry out steganalysis would order the palette to make their job easier. The palette from stegoed images will be compared with the palette of the original 256 colour image and the palette of a reduced colour unstegoed image to see how much the palette has changed. Finally the palette will be examined to look at how it changes when hiding long and short messages.

- Visual Inspection of the Image: This analysis method is based on evaluation through visual inspection of the image by independent evaluators. In all cases, the steganographic methods create a degree of distortion in the image, due to the reduction in the number of colours or the mixing and matching of existing colours. The question is how vulnerable are different techniques to detection through visual inspection of the image for tell tale distortion. An evaluator should typically look for a grainy structure or appearance; too few colours causing colour blocks and a lack of texture; lack of continuity in the colour and subtle distortions of any type 
For this evaluation 13 independent evaluators were used. All of the evaluators had a knowledge of computing and an understanding of how images are formed. However they did not have detailed knowledge of how steganography works. Each evaluator was provided with a brief description of steganography and told what features to look for in a stegoed picture. They were provided with a menu of folders and images and asked to identify the stegoed images (size 300 x 200 pixels). Each evaluator was presented with a suite of 18 folders. Each folder contained 6 images which were similar in content and lighting but were not identical. Each folder contained one stegoed image and five 256 colour unstegoed images. The first block of 6 folders thus had 6 stegoed images one for each steganography method under inspection (Stego1Bit, Stego2Bits, Stego3Bits, StegoColorCycle, StegoPRNG and StegoFridrich. Stego4Bits is not being evaluated here as it is clearly vulnerable to almost every type of inspection). Three sets of 6 folders were evaluated, a set of greyscale pictures of trees, an identical set of colour pictures of trees and a set of colour pictures of flowers.

- Low Level Visual Inspection of Image Pixels: This detection method is based on carrying out a detailed inspection of selected sections of an image at a high degree of magnification to determine whether anomalous patterns become apparent. As an inspection technique this might be problematical if the original cover image is not available for comparison. Under normal circumstances a person seeking to hide data in an image would be expected to use an image they have created themselves rather than a generally known image and therefore the original cover image would not be available for comparison with the stegoed image using this inspection method. The tell tale distortions which would typically be looked for include a lack of continuity where continuity is expected (Fridrich method) and a lack of texture where texture is expected (colour reduction).

The following shows the evaluation results for the evaluation methods described above.

\subsection{PATTERN ANALYSIS OF IMAGE PIXELS}

From the literature survey it is clear that Stego1Bit has a very high degree of vulnerability to steganalysis. This is due to the repeating patterns that are created in the least significant bit of the chosen colour. Thus this method is very vulnerable to detection from automated steganalysis methods. A variety of techniques of varying sophistication are used to combat 
this weakness. Stego2Bits, Stego3Bits, Stego4Bits and StegoColorCycle all break up the least significant bit pattern in different ways but are all extremely well known methods of hiding data. Therefore the patterns they create when attempting to hide the least significant bit pattern are only slightly more difficult to detect. For a method to be truly resilient against steganalysis using pattern searches bits must be hidden in an apparently random way. The pattern by which they are hidden must be reproducible but not easily reproducible. Thus the key based pseudo random number generation technique used by the Stego1BitPRNG method and the StegoFridrich method make these techniques highly resistant to this form of steganalysis.

\subsection{PATTERN ANALYSIS OF IMAGE PALETTE}

The pattern analysis of the image palettes will be carried out in two stages. First greyscale palettes will be examined and then colour palettes will be examined. In both cases the reduced unstegoed palettes will be shown and then the stegoed versions of these reduced palettes will be shown. A discussion of the results will also be included. When colours or grey levels are reduced for colour or greyscale images using colour reduction methods such as nearest colour and error diffusion, although the palette starts out with up to 256 colours or grey levels and for example 128 are required, the actual colour reduction level can be significantly less than the 128 colours or grey levels requested. The actual number of colours left depends on the original image used when reducing the colours or levels using the software Paint Shop Pro. Therefore although a reduction to 128 colours or grey levels is all that is required the images tested contained somewhat less than 128 colours / grey levels. Due to the manner in which the colour reduction is carried out by Paint Shop Pro it can take a bit of experimentation to get approximately the required number of colours / grey levels. Greyscale images seem to be more prone to overshooting the target when reducing colours than colour images are. The palettes have all been ordered by luminance in order to simplify the analysis of the contents.

\subsection{GREYSCALE IMAGES}

All of the steganography methods tested except StegoFridrich require a reduced palette to allow for the creation of new colours. The code used in this study embeds data in the least significant bits of red, green or blue values or a combination of these in all the methods tested except for StegoFridrich. Therefore although greyscale values are being examined in the first set of images, the red, green, blue values of pixel colours are still the location where the message bits are embedded. Shown below is a series of palettes for one particular image, first in greyscale and then in colour. Each palette shown is for the same image but the image in each case is composed of different numbers of greyscale levels or colours. Stego1Bit and 
Stego1BitPRNG require an image with a palette which is made up of a maximum of 128 colours. Stego2Bits and StegoColorCycle require an image with a palette with a maximum of 64 colours. Stego3Bits needs an image with a maximum of 32 colours and Stego4Bits requires an image with a palette with a maximum of 16 colours. StegoFridrich does not require any reduction in the number of colours in the image, as the method does not involve the creation of any new colours. Figure 5.1 shows the palette from the original greyscale image containing 249 levels of greyscale. Figure 5.2 and 5.3 show palettes for the same image whose colours have been reduced so that the image can be used to embed a message. Figure 5.1 is a typical greyscale palette but figure 5.2 and 5.3 clearly show a much-reduced range of greyscale levels. However the interesting thing discovered during this analysis is that because new greyscale levels are being created by the steganography process the palette fills up with or at least increases its number of greyscale levels to account for the new levels created. This results in the palettes looking much more similar to the original. Someone trying to carry out steganalysis won't see the original reduced palette, but the palette they will see will be very similar to a typical greyscale palette. The slight variations between original colours and newly created colours are not visually very discernible in these palettes as there is in any case a very gradual change between greyscale levels on a typical palette. The stegoed image palettes for the remaining steganography methods are also shown below in figures 5.4 to 5.8. Although they range from 138 to 218 greyscale levels they do not show the dramatic greyscale level reduction visible in the reduced palettes of the unstegoed images. Therefore creating an increased number of new levels of greyscale as a result of stegoing an image serves to make the palette look more like a typical palette which has somewhere close to 256 levels of greyscale.

There is however one giveaway factor which should be noted. If the RGB values are examined using for example the software Paint Shop Pro on a typical palette for greyscale the three RGB values are all the same for a given grey level. This was found for the palettes in figures 5.1, 5.2 and 5.3 below. However in all the stegoed images palettes, excluding StegoFridrich, when a pixel in the palette is passed over with the mouse the RGB values are shown and in some cases they are not the same for each colour in the palette. In figure 5.4 which shows the palette produced for an image following steganography using the Stego1Bit method, the blue value in the palette in some cases differs by one bit. The colours in the palette show that pixel index 103 is $87,87,86$. The LSB of the blue value has been changed. In the palette for Stego2Bits the value for the blue part of the RGB value can change by between 0 and 3 (the two least significant bits), using Stego3Bits it can change by between 0 and 7 and using Stego4Bits it can change by between 0 and 15 . The palette for the image 
produced following message embedding by the StegoColorCycle method could result in a one-bit change to any of the three RGB values in a colour. In figure 5.9, palette index 106 has a value of $87,86,86$. The LSB of the red value has been changed. These changes to the RGB values are immediately obvious in a greyscale picture when the RGB values are inspected as the different RGB combinations cannot exist in a greyscale image. In fact it is clearly no longer a greyscale image even though this is not obvious when looking at the image itself. The method StegoFridrich does not result in any changes to the original image palette and it also does not require any reduction in the number of colours. No tell tale signature about the fact that steganography has been carried out is therefore given away using this method. A large and a small message were embedded in the image using Stego1Bit to see what effect this had on the number of new colours produced and hence the appearance of the palette. The palettes are shown in figures 5.4 and 5.5. Figure 5.4 shows a palette produced by embedding a message which was 23 characters long. Figure 5.5 shows a palette produced by embedding a message which was the maximum size the image could hold. The palette in figure 5.5 more closely resembles the original palette in figure 5.1. Therefore embedding a larger message actually results in the palette more closely resembling a typical palette.

In conclusion it can be said that a visual inspection of the palette does not immediately show that steganography has been carried out on the image. In fact the bigger the message hidden in the image the less obvious it is. However an inspection of the precise RGB values which make up a range of greyscale levels shows a distinct telltale signature which is present in all methods which manipulate the pixel values. Only StegoFridrich is not vulnerable to this type of analysis.

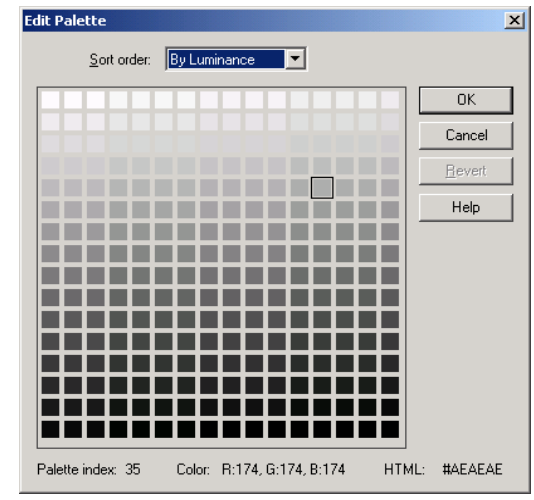

Figure 5.1 Palette for Greyscale (249 levels) levels)

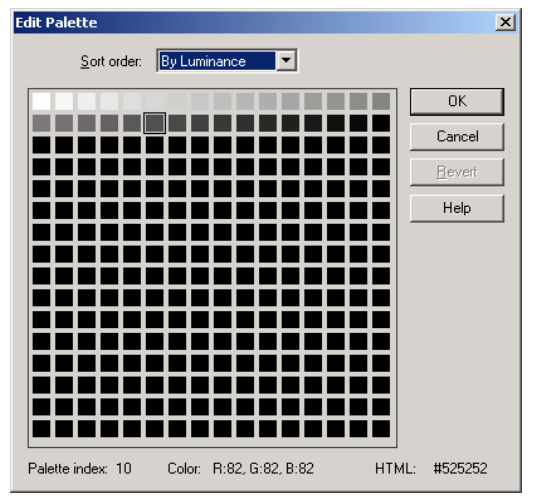

Figure 5.2 Palette for Greyscale (31 


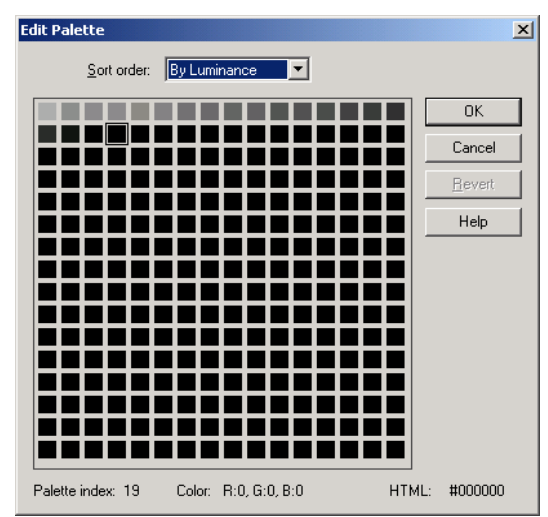

Figure 5.3 Greyscale (16 Levels)

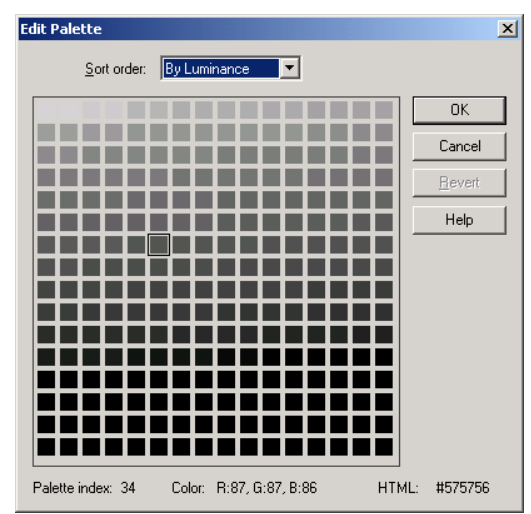

Figure 5.5 Palette for Stegoed Greyscale

Image 186 Levels using Stego1Bit

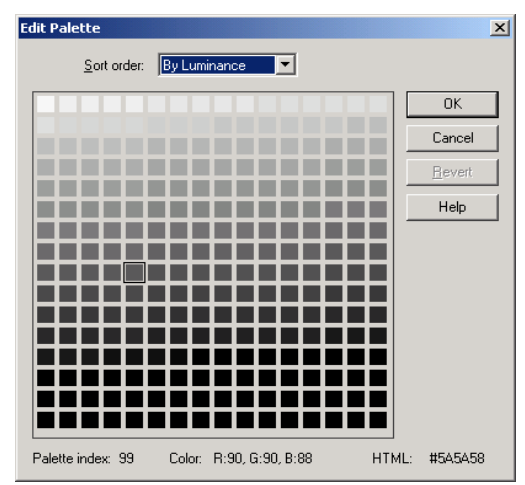

Figure 5.7 Palette for Stegoed Greyscale

Image with 200 Levels using Stego3Bits

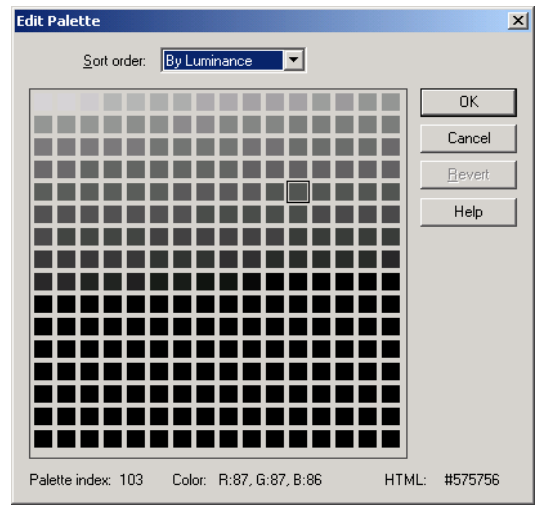

Figure 5.4 Stegoed Greyscale(138 levels)

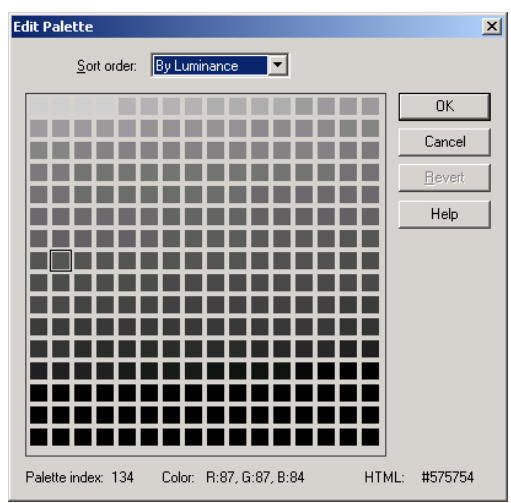

Figure 5.6 Palette for Stegoed Greyscale

Image with 205 Levels using Stego2Bit

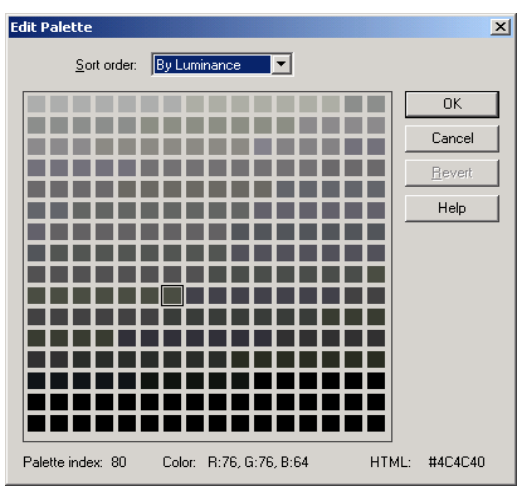

Figure 5.8 Palette for Stegoed Greyscale

Image with 218 Levels using Stego4Bits

\subsection{COLOUR IMAGES}

The code used in this study embeds data in the least significant bits of red, green or blue values or a combination of these in all the methods tested except for StegoFridrich. Thus all of the steganography methods tested except StegoFridrich require a reduced palette to allow 
for the creation of new colours in the palette. The first palette shown in figure 5.12 is a palette for a colour image which is the same image whose palettes were examined above in greyscale. It contains 256 colours. Two examples were taken of reduced palettes just to give a representation of what the reduced palettes look like. It was felt unnecessary to display all the reduced palettes. Figure 5.13 shows a palette whose colours have been reduced to 128 . This is required for the Stego1Bit and Stego1BitPRNG methods. Although it is obvious that about half the possible colours are being used this does not mean that they were necessarily reduced for stegoing, although it is a pointer. The key point is that if a steganographer uses their own unique images the stegoanalyst should not have access to the original unstegoed image and correspondingly will never see these reduced palettes. Figure 5.14 shows the palette used for the Stego4Bits steganography method. It only has 16 different colours.

We stegoed images using Stego1Bit with a message less than the size of the image and a message the capacity of the image. In their unordered form these palettes looked like any other image palettes. But when they have been ordered by luminance a pattern is visible. What it shows is that some of the colours are arranged in pairs of very similar colours. This occurs because the LSB of some colours have been changed resulting in a very similar new colour. This new colour may not be visible when examining the image but it is a new colour and must be listed separately in the palette. This is a pointer to the occurrence of steganography. The maximum size message has resulted in this case in the creation of extra new colours. This fills up the unordered palette which tends to hide the fact that steganography has occurred but as it also creates more pairings when the palette is ordered which does highlight the fact that steganography has been carried out. Figure 5.22 shows a palette from an image produced by stegoing with Stego1Bit PRNG. Pairs of colours are visible in the palette in the same way as they are visible in the palette for Stego1Bit. It does not follow that because the palette is a pointer to steganography that the stegoanalyst will be able to read the message from the image. In the case of Stego1BitPRNG the message bits are pseudo randomly spread over the image pixels. Therefore the key to the pseudo random number generator would need to be known as well as the algorithm. Unfortunately though if a stegoanalyst is looking for stegoed images patterns in the palette will result in the image being carefully analysed defeating the purpose of steganography which is to prevent anyone knowing that a message is being sent at all. Unlike a greyscale image, if the RGB values are examined using for example Paint Shop Pro software on a typical palette for a colour image this time the three RGB values are not the same for a given colour unless it is a greyscale colour. In a greyscale image they are the same. This is an advantage for colour images because changing LSBs in therefore not as noticeable. However as discussed above following 
steganography with all the methods tested here except StegoFridrich groups of very similar colours are produced. If the first group in Stego4Bits is examined the RGB values for the pale green colour are as follows:
$192,220,206$
$192,220,203$
$192,220,202$
$192,220,201$
$192,220,199$
$192,220,198$
$192,220,196$
$192,220,194$

192, 220, 192

Therefore the fact that the 4 LSBs of the blue values have been changed is clearly visible when the RGB values are read using Paint Shop Pro. When StegoColorCycle is used any of the three RGB values can be changed. The first 4 colours in the palette are a group (pale blue) and have the following values 191, 209, 215, 191, 209, 214, 190, 209, 215 and 191, 208, 215. In the second colour, blue has been changed, in the third colour red has been changed and in the fourth colour green has been changed. The palette produced when the image was stegoed by the StegoFridrich method gives nothing away about the fact that steganography has been carried out because the palette remains exactly the same as the palette for the original image. As a final observation it is arguably true to say that, in the case of methods which use reduced palettes, although both colour and greyscale palettes show the same tell tale signs that steganography has been carried out it does seem to be harder to visually see the clusters of similar colours or grey levels in the greyscale palette. Again however looking at the colour/greyscale RGB values in detail (the RGB integer values) clearly shows patterns produced in the RGB values following steganography.

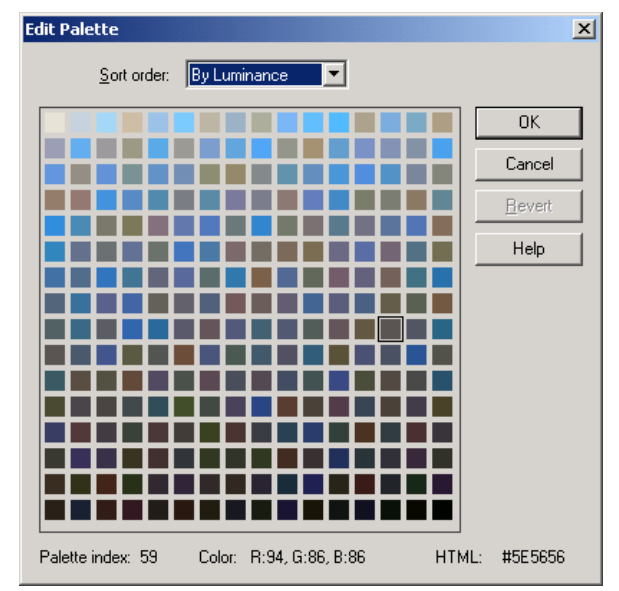

Figure 5.12 Palette with 256 colours

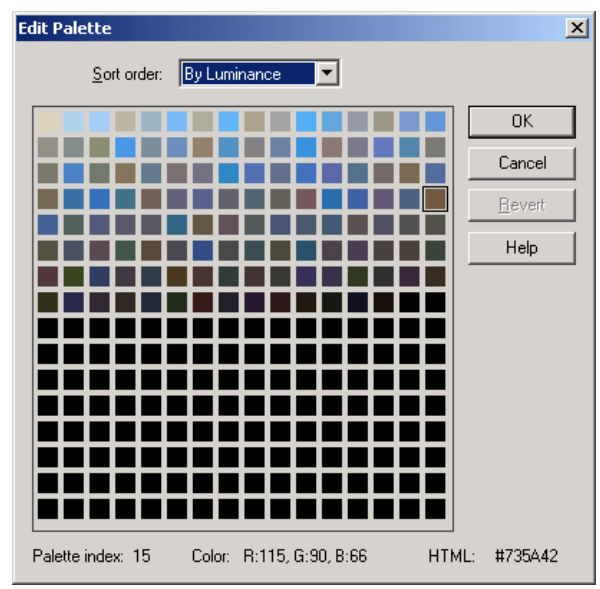

Figure 5.13 Palette with 128 colours 


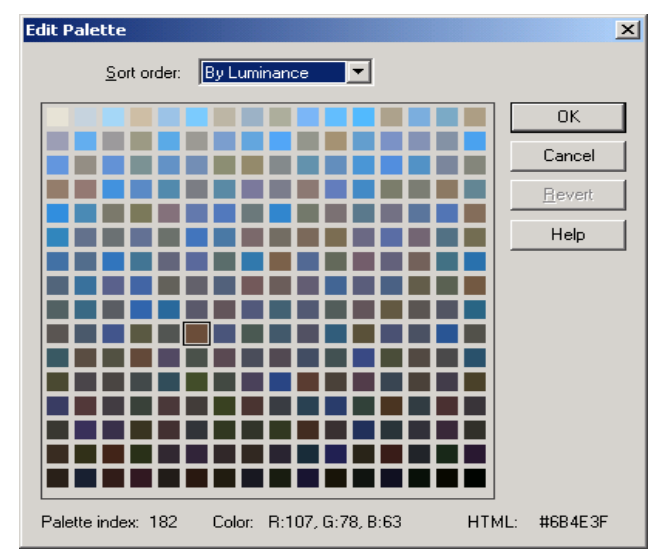

Figure 5.22 Palette for Colour Image with
256 Colours Stegoed using Stego1Bit

\subsection{VISUAL INSPECTION OF THE IMAGE}

This evaluation method was based on visual inspection of the image by 13 independent evaluators. The question is how vulnerable are different steganographic techniques to detection through visual inspection of the image for tell tale distortion. All of the evaluators had knowledge of computing and an understanding of how images are formed. However they did not have detailed knowledge of how steganography works. Each evaluator was provided with a brief description of steganography and told what features to look for in a stegoed picture. They were provided with a menu of folders and images and asked to identify the stegoed images. Each evaluator was presented with a suite of 18 folders. Each folder contained 6 images which were similar in content and lighting but were not identical. The evaluators were informed that each folder contained one stegoed image and five 256-colour unstegoed images. The first block of 6 folders thus had 6 stegoed images one for each steganography method under inspection (Stego1Bit, Stego2Bits, Stego3Bits, StegoColorCycle, StegoPRNG and StegoFridrich. Stego4Bits is not being evaluated here, as it is clearly vulnerable to almost every type of inspection). Three sets of 6 folders were evaluated, a set of greyscale pictures of trees, an identical set of colour pictures of trees and a set of colour pictures of flowers. The results are shown below. Please see Appendix $\mathrm{C}$ for the instructions given to the evaluators and copies of the stegoed images. Each folder contained 6 images and there were 13 evaluators. Therefore completely random selection of the images would yield a spread of about $16 \%$ per image. In Table 5.1 below the percentage selection of the stegoed images in each folder has a spread of $15 \%, 0 \%, 0 \%, 31 \%, 23 \%$ and $8 \%$. This is quite an uneven detection rate however even more surprising is that Stego1Bit was selected $15 \%$ of the time while Stego2Bits and Stego3Bits which should be easier to detect were not selected at all. StegoColorCycle was chosen $31 \%$ of the time which is twice 
what an average unguided distribution would be and this corresponds to our expectation that as a stegoed 64 colour image it should be relatively easy to detect. Stego1BitPRNG was chosen $23 \%$ of the time and StegoFridrich was chosen $8 \%$ of the time.

\begin{tabular}{|c|c|c|c|}
\hline Folder & Picture & Stego Method & Percentage Chosen \\
\hline \multirow[t]{6}{*}{ BW Tree Folder 1} & BW_Tree1 & & $8 \%$ \\
\hline & BW Tree2 & & $54 \%$ \\
\hline & BW_Tree3 & Stego1Bit & $15 \%$ \\
\hline & BW Tree4 & & $8 \%$ \\
\hline & BW Tree5 & & $15 \%$ \\
\hline & BW_Tree6 & & $0 \%$ \\
\hline \multirow[t]{6}{*}{ BW Tree Folder 2} & BW Tree1 & & $0 \%$ \\
\hline & BW Tree2 & & $15 \%$ \\
\hline & BW Tree3 & & $39 \%$ \\
\hline & BW Tree4 & Stego2Bits & $0 \%$ \\
\hline & BW Tree5 & & $15 \%$ \\
\hline & BW Tree6 & & $31 \%$ \\
\hline \multirow[t]{6}{*}{ BW Tree Folder 3} & BW_Tree1 & Stego3Bits & $0 \%$ \\
\hline & BW Tree2 & & $31 \%$ \\
\hline & BW Tree3 & & $15 \%$ \\
\hline & BW Tree4 & & $0 \%$ \\
\hline & BW_Tree5 & & $31 \%$ \\
\hline & BW Tree6 & & $23 \%$ \\
\hline \multirow[t]{6}{*}{ BW Tree Folder 4} & BW_Tree1 & & $31 \%$ \\
\hline & BW Tree2 & StegoColorCycle & $31 \%$ \\
\hline & BW Tree3 & & $23 \%$ \\
\hline & BW Tree4 & & $0 \%$ \\
\hline & BW Tree5 & & $15 \%$ \\
\hline & BW Tree6 & & $0 \%$ \\
\hline \multirow[t]{6}{*}{ BW Tree Folder 5} & BW Tree1 & & $23 \%$ \\
\hline & BW Tree2 & & $23 \%$ \\
\hline & BW Tree3 & & $8 \%$ \\
\hline & BW_Tree4 & & $15 \%$ \\
\hline & BW Tree5 & Stego1BitPRNG & $23 \%$ \\
\hline & BW_Tree6 & & $8 \%$ \\
\hline \multirow[t]{5}{*}{ BW Tree Folder 6} & BW Tree1 & & $8 \%$ \\
\hline & BW Tree2 & & $8 \%$ \\
\hline & BW_Tree3 & & $31 \%$ \\
\hline & BW Tree5 & & $31 \%$ \\
\hline & BW_Tree6 & StegoFridrich & $8 \%$ \\
\hline
\end{tabular}

Table 5.1 Visual Inspection of Black and White Images of Trees

It is difficult to determine with any degree of certainty that any of these detection rates indicate the evaluators were able to detect the presence of a stegoed image. This is particularly true because in many cases unstegoed images were selected $31 \%$ of the time. BW_Tree2 in BW Tree Folder 1 which was not stegoed was chosen 54\% of the time which is $23 \%$ more than any of the stegoed images. The most likely explanation is that users were looking for unexpected features in the images and were finding unexpected features which were not related to the steganography process. 


\begin{tabular}{|c|c|c|c|}
\hline Folder & Picture & Stego Method & Percentage Chosen \\
\hline \multirow[t]{6}{*}{ Colour Tree Folder 1} & Colour Tree1 & & $15 \%$ \\
\hline & Colour Tree 2 & Stego1BitPRNG & $46 \%$ \\
\hline & Colour Tree 3 & & $8 \%$ \\
\hline & Colour Tree4 & & $8 \%$ \\
\hline & Colour Tree5 & & $15 \%$ \\
\hline & Colour Tree6 & & $8 \%$ \\
\hline \multirow[t]{6}{*}{ Colour Tree Folder 2} & Colour Tree1 & & $15 \%$ \\
\hline & Colour Tree2 & & $23 \%$ \\
\hline & Colour Tree3 & & $8 \%$ \\
\hline & Colour Tree4 & Stego2Bits & $39 \%$ \\
\hline & Colour Tree5 & & $15 \%$ \\
\hline & Colour Tree6 & & $0 \%$ \\
\hline \multirow[t]{6}{*}{ Colour Tree Folder 3} & Colour Tree 1 & & $0 \%$ \\
\hline & Colour Tree2 & & $15 \%$ \\
\hline & Colour Tree3 & & $8 \%$ \\
\hline & Colour Tree4 & & $8 \%$ \\
\hline & Colour Tree5 & & $8 \%$ \\
\hline & Colour Tree6 & Stego3Bits & $62 \%$ \\
\hline \multirow[t]{6}{*}{ Colour Tree Folder 4} & Colour Tree1 & StegoColorCycle & $54 \%$ \\
\hline & Colour Tree2 & & $8 \%$ \\
\hline & Colour Tree3 & & $8 \%$ \\
\hline & Colour Tree4 & & $23 \%$ \\
\hline & Colour Tree5 & & $8 \%$ \\
\hline & Colour Tree6 & & $0 \%$ \\
\hline \multirow[t]{6}{*}{ Colour Tree Folder 5} & Colour Tree1 & & $0 \%$ \\
\hline & Colour Tree2 & & $8 \%$ \\
\hline & Colour Tree3 & Stego1Bit & $8 \%$ \\
\hline & Colour Tree4 & & $23 \%$ \\
\hline & Colour Tree5 & & $31 \%$ \\
\hline & Colour Tree6 & & $31 \%$ \\
\hline \multirow[t]{6}{*}{ Colour Tree Folder 6} & Colour Tree 1 & & $15 \%$ \\
\hline & Colour Tree2 & & $8 \%$ \\
\hline & Colour Tree3 & & $15 \%$ \\
\hline & Colour Tree4 & & $15 \%$ \\
\hline & Colour Tree5 & StegoFridrich & $31 \%$ \\
\hline & Colour Tree6 & & $15 \%$ \\
\hline
\end{tabular}

Table 5.2 Visual Inspection of Colour Images of Trees

In table 5.2 above there is a spread of $46 \%, 39 \%, 62 \%, 54 \%, 8 \%$ and $31 \%$ for selection of the stegoed images. The highest selection percentage for an unstegoed image is $31 \%$. This immediately suggested that the evaluators were significantly more successful at detecting steganography in coloured images than they were with the grey level images in table 5.1. Stego2bits, Steg3Bits and StegoColorCycle have high detection rates which are broadly in line with what one might expect in that more evaluators detected Stego3Bits than detected Stego2Bits. The detection rate for Stego1Bit was quite low. Again however unexpected results are seen. Stego1BitPRNG should not show a more visible distortion than Stego1Bit however it appears to be 6 times easier to detect. These results how that colour reduction is more detectable in colour images than in grey level images however it is difficult to be certain how much the evaluators were misled by possible unexpected features in the test images 
which were not related to steganography.

\begin{tabular}{|c|c|c|c|}
\hline Folder & Picture & Stego Method & Percentage Chosen \\
\hline \multirow[t]{6}{*}{ Colour Flower Folder 1} & Colour Flower1 & & $23 \%$ \\
\hline & Colour Flower2 & & $8 \%$ \\
\hline & Colour Flower3 & Stego1Bit & $31 \%$ \\
\hline & Colour Flower4 & & $15 \%$ \\
\hline & Colour Flower5 & & $8 \%$ \\
\hline & Colour Flower6 & & $15 \%$ \\
\hline \multirow[t]{6}{*}{ Colour Flower Folder 2} & Colour Flower1 & & $15 \%$ \\
\hline & Colour Flower2 & & $23 \%$ \\
\hline & Colour Flower3 & & $8 \%$ \\
\hline & Colour Flower4 & & $23 \%$ \\
\hline & Colour Flower5 & Stego2Bits & $15 \%$ \\
\hline & Colour Flower6 & & $15 \%$ \\
\hline \multirow[t]{6}{*}{ Colour Flower Folder 3} & Colour Flower1 & & $8 \%$ \\
\hline & Colour Flower2 & & $8 \%$ \\
\hline & Colour Flower3 & Stego3Bits & $54 \%$ \\
\hline & Colour Flower4 & & $0 \%$ \\
\hline & Colour Flower5 & & $0 \%$ \\
\hline & Colour Flower6 & & $23 \%$ \\
\hline \multirow[t]{6}{*}{ Colour Flower Folder 4} & Colour Flower1 & & $23 \%$ \\
\hline & Colour Flower2 & & $15 \%$ \\
\hline & Colour Flower3 & & $0 \%$ \\
\hline & Colour Flower4 & StegoColorCycle & $31 \%$ \\
\hline & Colour Flower5 & & $8 \%$ \\
\hline & Colour Flower6 & & $23 \%$ \\
\hline \multirow[t]{6}{*}{ Colour Flower Folder 5} & Colour Flower1 & & $23 \%$ \\
\hline & Colour Flower2 & & $8 \%$ \\
\hline & Colour Flower3 & & $15 \%$ \\
\hline & Colour Flower4 & & $8 \%$ \\
\hline & Colour Flower5 & & $31 \%$ \\
\hline & Colour Flower6 & Stego1BitPRNG & $15 \%$ \\
\hline \multirow[t]{6}{*}{ Colour Flower Folder 6} & Colour Flower1 & StegoFridrich & $23 \%$ \\
\hline & Colour Flower2 & & $0 \%$ \\
\hline & Colour Flower3 & & $31 \%$ \\
\hline & Colour Flower4 & & $15 \%$ \\
\hline & Colour Flower5 & & $31 \%$ \\
\hline & Colour Flower6 & & $0 \%$ \\
\hline
\end{tabular}

\section{Table 5.3 Visual Inspection of Colour Images of Flowers}

In table 5.3 above there is a spread of $31 \%, 15 \%, 54 \%, 31 \%, 15 \%$ and $23 \%$ for selection of the stegoed images. As with Table 5.2 above the highest selection percentage for an unstegoed image is $31 \%$. Some of the results are in line with expectations. Stego3Bits has a high detection rate at $54 \%$. This contrasts sharply with the highest selection rate of $31 \%$ for an unstegoed image. However Stego1Bit has a detection rate twice as high as Stego2Bits. Stego1BitPRNG as might have been expected has a low detection rate. As with the two previous tables it is difficult to be certain how much the evaluators were misled by possible unexpected features in the test images which were not related to steganography. 


\subsection{LOW LEVEL VISUAL INSPECTION OF IMAGE PIXELS}

This section of the analysis deals with the low level visual inspection of selected parts of the images. Although some of the images are shown at magnification $\mathrm{x} 3$, most are at the maximum magnification of 32 which is satisfactory for viewing individual pixels. In order to make the analysis more meaningful all magnification $x 3$ images show the same part of the same image and magnification $\times 32$ images show the same part of the same image. Figure 5.23 and figure 5.24 which are black and white images of trees at magnification $\times 3$ show the original 256 grey level image and the same image reduced to 128 grey levels and then stegoed using Stego1Bit. Some slight differences in shading are visible when both the original and the stegoed images are placed side by side. However it is unlikely that the distortion in the stegoed image would be detectable without the original to compare it with. The same observations could be made in the case of the original and stegoed magnification $\mathrm{x} 3$ colour images in figures 5.32 and 5.33 .

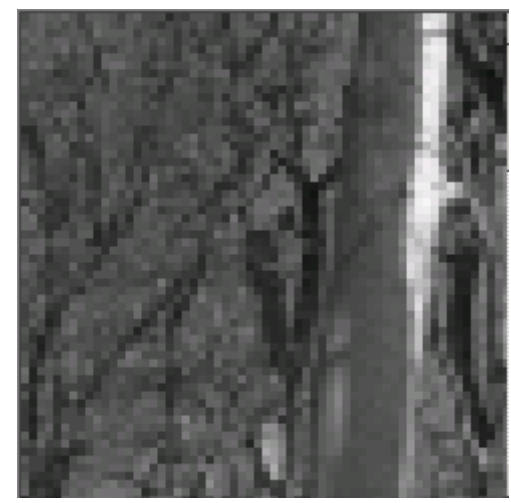

Figure 5.23 Original Black and White Tree (Magnification x3)

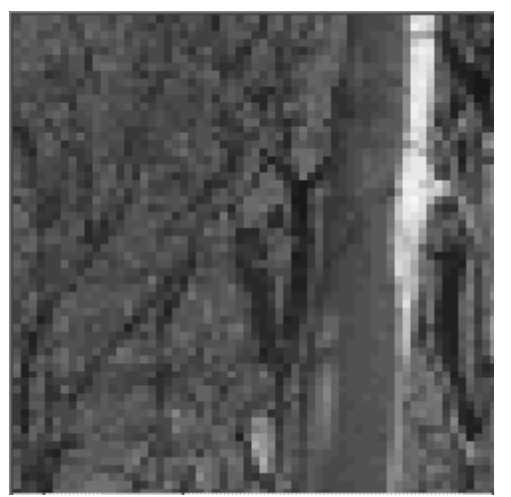

Figure 5.24 Stego1Bit Black and White Tree (Magnification x3)

An examination of the original black and white tree at magnification x 32 showed a highly fragmented image segment with a high degree of texture as seen by the rapid variations in pixel grey levels. As the number of grey levels is reduced for other steganography methods the amount of texture decreases slightly. It is only the 16 grey level images which showed suspiciously large continuous blocks of pixels. This observation is also true of the unmagnified images in that it is only the 16 grey levels image which clearly stands out as being artificially modified. Interestingly the grey level variations added by the steganography process do not appear, except in the case of stego4Bits, to add significant texture back into the images. This is because the additional colours / grey levels produced by the stegoing process are insufficiently different to be easily detected through visual inspection when only the three least significant bits of the blue part of the RGB colour are being modified. This can clearly be seen by inspecting the magnification x 32 images. 


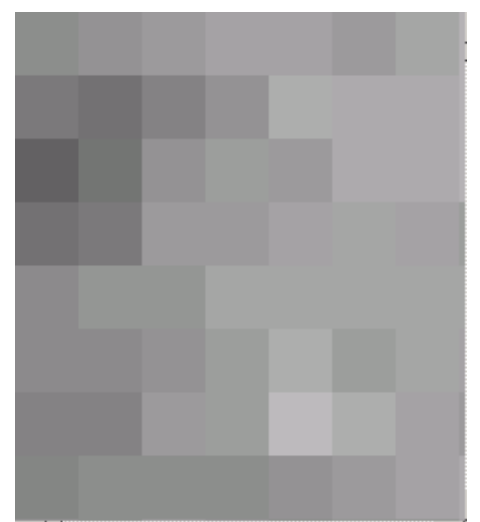

Figure 5.25 Original Black and

White Tree (Magnification x32)

It might have been expected that a detailed inspection of the pixels in an image which has been stegoed using stegoFridrich would show a high degree of texture and a lack of continuity in the pixels. However as can seen from figure 5.30 and figure 5.31 below this does not appear to be the case looking at the images used for this evaluation. This is because the original image is itself highly fragmented and colour substitution is based on shortest distance so the magnified stegoed image seems unremarkable.

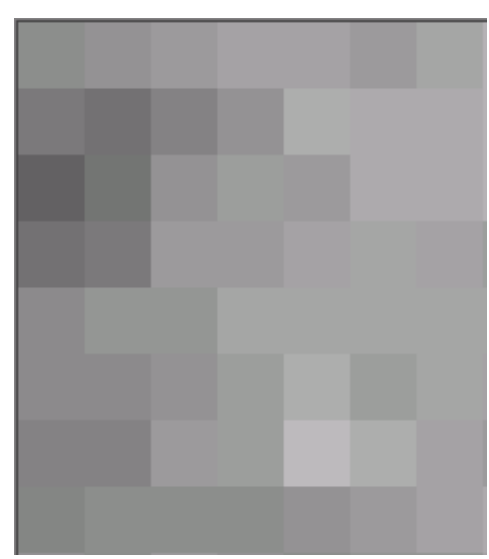

Figure 5.30 256 Grey Levels Black \& White Tree (Magnification x32)

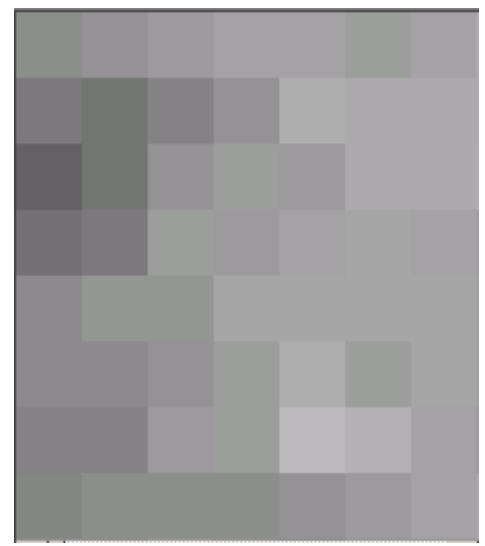

Figure 5.31 StegoFridrich Black \& White Tree (Magnification x32)

Figure 5.32 and figure 5.33 which are colour images of trees at magnification $\times 3$ show the original 256 colour image and the same image reduced to 128 colours and then stegoed using Stego1Bit. As with the black and white images above some slight differences in shading are visible when both the original and the stegoed images are placed side by side and compared. However as above it is unlikely that the distortion in the stegoed image would be detectable without the original to compare it with. 


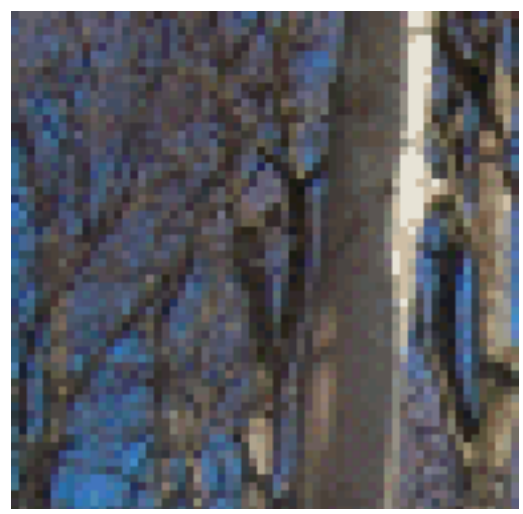

Figure 5.32 Original Tree (Magnification x3)

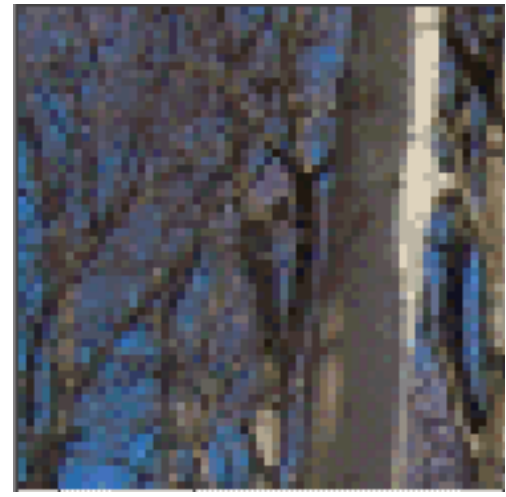

Figure 5.33 Stego1Bit Tree (Magnification x3)

An examination of the original colour tree at magnification x32 (figure 5.34) shows a highly fragmented image segment with a high degree of texture as seen by the rapid variations in pixel colours. As the numbers of colours are reduced for other stego methods the amount of texture decreases more rapidly than seems to be the case for the grey scale images. The 16 colour image shows suspiciously large continuous blocks of pixels but the 64 and 32 colour images also looked quite blocky. As with the grey scale images the colour variations added by the steganography process do not appear, except in the case of stego4Bits to add significant texture back into the images.

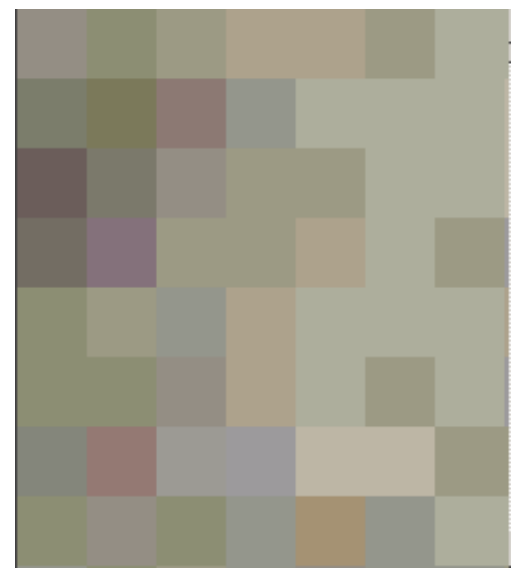

\section{Figure 5.34 Original Tree} (Magnification x32)

As was stated above it might have been expected that a detailed inspection of the pixels in an image which has been stegoed using StegoFridrich would show an excessive degree of texture and lack of continuity in the pixels. However this was not true in the case of the grey scale images and as can be seen in figure 5.39 and figure 5.40 below this is not the case in the colour image which has been examined to date in this section. It would appear that StegoFridrich is only vulnerable to this form of analysis if it is implemented on an image which has an area with a high degree of continuity in its pixels. Figure 5.41 shows a scene 
which appears to have a highly continuous block of white colour. Because, in this particular image the closest colour in the palette is a grey $(224,224,224)$ StegoFridrich yields a highly distorted stegoed image in figure 5.42. This can be seen in more detailed in the magnified sections of the white background in figure 5.43 and 5.44. Conversely methods such as Stego1Bit lend themselves to images with blocks of continuous colour. Thus the steganographer must take reasonable precautions to ensure that the image they choose suits the type of steganography they wish to carry out.

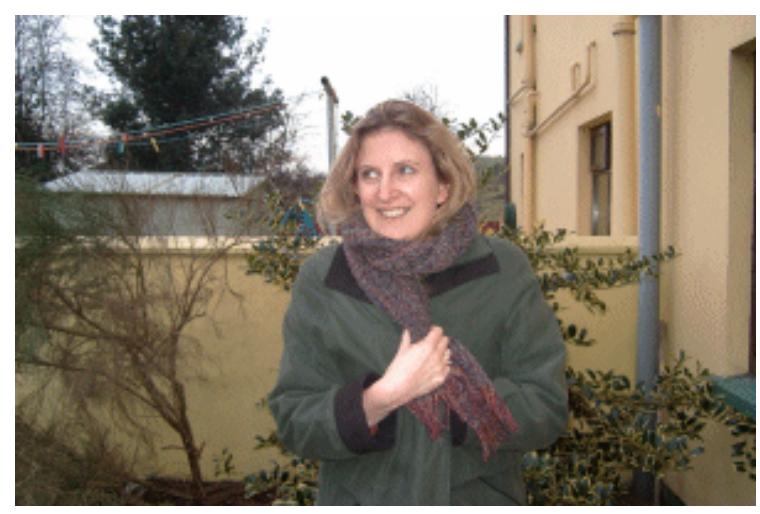

Figure 5.41 256 Colour Karen

\section{Figure 5.43 256 Colour Karen} (Magnification x32)

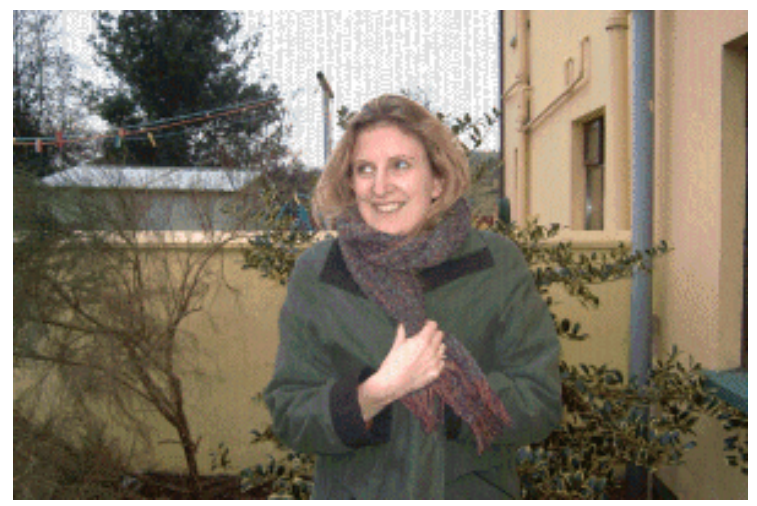

Figure 5.42 StegoFridrich Karen
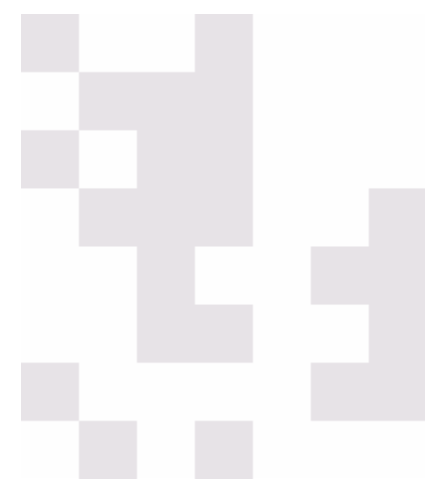

Figure 5.44 StegoFridrich Karen (Magnification x32)

Figure 5.45 shows an image of a flower which was used in this study. Figure 5.46 shows the same image magnified three times and figure 5.47 shows the image magnified 3 times following steganography with the stgeoFridrich method. Comparing 5.46 and 5.47 it can be seen that there is increased noise in the stegoed image. This in itself does not necessarily indicate the existence of steganography to an analyst. Figure 5.48 shows the same image decreased to 128 colours. We also reduced the colour flower after a message had been inserted using stego1Bit. There is no distinguishable difference between the two 
images at all. But if the reduced colour image is compared with the original in figure 5.46 it could be seen that some of the colour is lost from the centre of the flower and the image overall has a more noisy appearance. However without the benefit of the original image these artefacts would not necessarily lead to the conclusion that steganography had been carried out on these images but simply indicate that they were of a poorer quality than they might be and have a higher noise level.

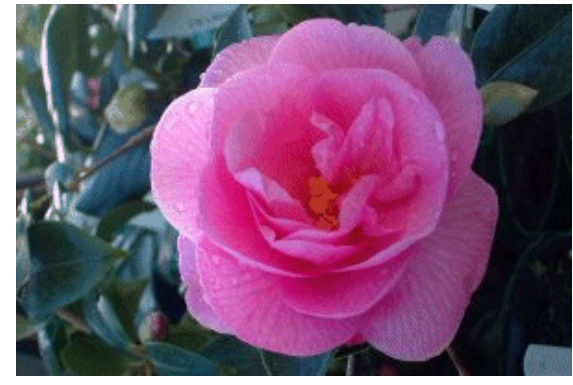

Figure 5.45 Original Flower

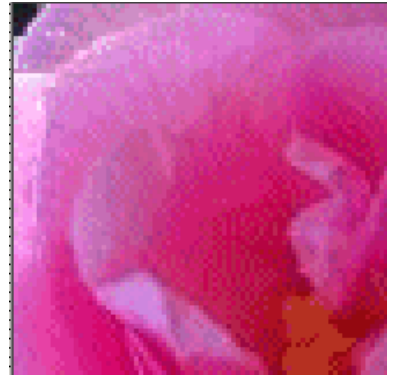

Figure 5.46 Flower x3

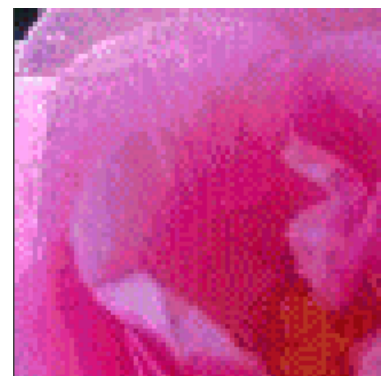

Figure 5.47 StegoFridrich $x 3$

\subsection{Evaluation Conclusion}

All of the steganography methods tested except StegoFridrich require a reduced palette to allow for the creation of new colours. The code used in this study embeds data in the least significant bits of red, green or blue values or a combination of these in all the methods tested except for StegoFridrich. Therefore although greyscale values are being examined in the first set of images, the red, green, blue values of pixel colours are still the location where the message bits are embedded. The interesting thing discovered during this analysis is that because new greyscale levels are being created by the steganography process the palette fills up with or at least increases its number of greyscale levels to account for the new levels created. This results in the palettes looking much more similar to the original. Someone trying to carry out steganalysis will not see the original reduced palette, but the palette they will see will be very similar to a typical greyscale palette. The slight variations between original colours and newly created colours are not visually very discernible in these palettes as there is in any case a very gradual change between greyscale levels on a typical palette. Although they range from 138 to 218 greyscale levels they do not show the dramatic greyscale level reduction visible in the reduced palettes of the unstegoed images. Therefore creating an increased number of new levels of greyscale as a result of stegoing an image serves to make the palette look more like a typical palette which has somewhere close to 256 levels of greyscale. There is however one giveaway factor which should be noted. If the RGB values are examined using for example the software Paint Shop Pro on a typical palette for greyscale 
the three RGB values are all the same for a given greylevel. This was found for the palettes in figures 5.1, 5.2 and 5.3 below. However in all the stegoed images palettes, excluding StegoFridrich, when a pixel in the palette is passed over with the mouse the RGB values are shown and in some cases they are not the same for each colour in the palette. In one of our palette results produced for an image following steganography using the Stego1Bit method, the blue value in the palette in some cases differs by one bit. The colours in the palette show that pixel index 103 is $87,87,86$. The LSB of the blue value has been changed. In the palette for Stego2Bits the value for the blue part of the RGB value can change by between 0 and 3 (the two least significant bits), using Stego3Bits it can change by between 0 and 7 and using Stego4Bits it can change by between 0 and 15 . The palette for the image produced following message embedding by the StegoColorCycle method could result in a one-bit change to any of the three RGB values in a colour. For instance, palette index 106 has a value of 87, 86, 86. The LSB of the red value has been changed. These changes to the RGB values are immediately obvious in a greyscale picture when the RGB values are inspected as the different RGB combinations cannot exist in a greyscale image. In fact it is clearly no longer a greyscale image even though this is not obvious when looking at the image itself.

\begin{tabular}{|c|c|c|c|c|c|c|c|c|}
\hline & Rating & $\begin{array}{l}1 \\
\text { Bit }\end{array}$ & $\begin{array}{l}2 \\
\text { Bits } \\
\end{array}$ & $\begin{array}{l}3 \\
\text { Bits } \\
\end{array}$ & $\begin{array}{l}4 \\
\text { Bits } \\
\end{array}$ & $\begin{array}{l}\text { Color } \\
\text { Cycle } \\
\end{array}$ & $\begin{array}{l}\text { 1Bit } \\
\text { prng }\end{array}$ & $\begin{array}{l}\text { Stego } \\
\text { Fridrich }\end{array}$ \\
\hline \multirow{3}{*}{$\begin{array}{l}\text { Pattern } \\
\text { Analysis of } \\
\text { the Bits }\end{array}$} & Not Vulnerable & & & & & & $x$ & $x$ \\
\hline & $\begin{array}{l}\text { Somewhat } \\
\text { Vulnerable }\end{array}$ & & $x$ & $x$ & $x$ & $x$ & & \\
\hline & Very Vulnerable & $x$ & & & & & & \\
\hline \multirow{3}{*}{$\begin{array}{l}\text { Pattern } \\
\text { Analysis of } \\
\text { the Palette }\end{array}$} & Not Vulnerable & & & & & & & $x$ \\
\hline & $\begin{array}{l}\text { Somewhat } \\
\text { Vulnerable } \\
\end{array}$ & & & & & & & \\
\hline & Very Vulnerable & $x$ & $x$ & $x$ & $x$ & $x$ & $x$ & \\
\hline \multirow{3}{*}{$\begin{array}{l}\text { Visual } \\
\text { Inspection } \\
\text { of the Image }\end{array}$} & Not Vulnerable & & & & & & & $x$ \\
\hline & $\begin{array}{l}\text { Somewhat } \\
\text { Vulnerable } \\
\end{array}$ & $x$ & $\mathrm{x}$ & & & & $x$ & \\
\hline & Very Vulnerable & & & $x$ & $x$ & $x$ & & \\
\hline \multirow{3}{*}{$\begin{array}{l}\text { Detailed } \\
\text { Visual } \\
\text { Inspection } \\
\text { of the Image } \\
\text { Pixels }\end{array}$} & Not Vulnerable & $x$ & $x$ & & & & $x$ & \\
\hline & $\begin{array}{l}\text { Somewhat } \\
\text { Vulnerable }\end{array}$ & & & $x$ & & $\mathrm{X}$ & & $x$ \\
\hline & Very Vulnerable & & & & $x$ & & & \\
\hline
\end{tabular}

\section{Table 1: Summary of Evaluation Results}

The method StegoFridrich does not result in any changes to the original image palette and it also does not require any reduction in the number of colours. No tell tale signature about the 
fact that steganography has been carried out is therefore given away using this method. A large and a small message were embedded in the image using Stego1Bit to see what effect this had on the number of new colours produced and hence the appearance of the palette. Therefore embedding a larger message actually results in the palette more closely resembling a typical palette. In conclusion it can be said that a visual inspection of the palette does not immediately show that steganography has been carried out on the image. In fact the bigger the message hidden in the image the less obvious it is. However an inspection of the precise RGB values which make up a range of greyscale levels shows a distinct telltale signature which is present in all methods which manipulate the pixel values. Only StegoFridrich is not vulnerable to this type of analysis.

Table 1 shows a summary of the evaluation results presented previously. While some of the results presented previously can be considered to be a little imprecise due to ambiguity in the findings, the effect of unexpected but natural artefacts in images, it is reasonable to draw approximate conclusions with the information available. StegoFridrich while having some weaknesses particularly where the image being used has not been chosen carefully appears to be the most secure method by a significant margin. All of the other methods suffer from an underlying weakness in that the requirement for colour reduction and replacement leaves a very strong steganography signature in the palette. After StegoFridrich, Stego1BitPRNG and Stego1Bit are the next most secure methods in that order. The strengths of StegoColorCycle in terms of having a degree of resistance to pattern analysis of the least significant bits appears to be cancelled out by degree of colour reduction required. Finally despite the additional data hiding capacity of Stego2Bits, Stego3Bits and Stego4Bits the security of the steganography method being used decreases as the data hiding capacity increases making these progressively less desirable techniques.

\section{CONCLUSION}

As all the of the methods evaluated required either colour reduction of the original images palette or colour substitution in the stegoed image, they all had their own weaknesses as the stegoed image inevitably suffered some distortion from the steganography process. In the case of colour reduction based techniques there were strong tell-tail signs in the palette as well. Overall the colour rearrangement technique appeared to be the most resistant to detection as long as suitable images were chosen. Techniques that attempt to maximise the message size that they can store; appear to be the least resistant to detection. 


\section{REFERENCES}

[Brown94]Brown A., S-Tools for Windows, Shareware, 1994.

ftp://idea.sec.dsi.unimi.it/pub/security/crypt/code/s-tools3.zip(version 3)

[Day98] Day Bill and Knudsen Jonathan, Image Processing with Java 2D www.javaworld.com/javaworld/jw-09-1998/jw-09-media p.html, August 1998.

[Efford00] Efford, Nick, Digital Image Processing a Practical Introduction using Java, Addison-Wesley, 2000.

[Fridrich99] Fridrich Jiri, A New Steganographic Method for Palette-Based Images. Center for Intelligent Systems, SUNY Binghamton, Binghamton, New York. IS\&T's PICS Conference, pp.285-289, 1999.

[Fridrich00] Fridrich Jiri, Rui Du, Secure Steganographic Methods for Palette Images. Center for Intelligent Systems, Dept. of SS\&IE, SUNY Binghamton, Binghamton, New York. Information Hiding, Third International Workshop, IH'99 Dresden Germany, September / October Proceedings, Computer Science 1768. pp. 47-60, 2000.

[Hansmann96] Hansmann F. Steganos. Deus Ex Machina Communications. http://www.steganography.com 1996

[Johnson98] Johnson Neil F., Sushil Jajodia, Steganalysis of Images Created Using Current Steganography Software, Centre for Secure Information Systems, George Mason University, Fairfax, Virginia, Information Hiding, Second International Workshop, IH'98 Portland, Oregon, USA, pp. 273-289, April 1998.

[Johnson01] Johnson Neil F., Zoran Duric, Sushil Jajodia, Information Hiding, and Watermarking - Attacks \& Countermeasures, Kluwer 2001.

[Murray96] Murray James D., William van Ryper, Encyclopedia of Graphics File Formats, Second Edition, O'Reilly and Associates Inc., 1996

[Pfitzmann96] Pfitzmann Birgit. Information Hiding Terminology. First International Workshop, Cambridge, UK, Proceedings, Computer Science 1174. pp. 347-350, May -June www.ijde.org 
1996.

[Wayner02] Wayner Peter, Disappearing Cryptography, Information Hiding: Steganography and Watermarking, $2^{\text {nd }}$ Edition, Morgan Kaufmann, 2002.

[Westfield99] Westfield Andreas and Andreas Pfitzmann, Attacks on Steganographic Systems. Third International Workshop, IH'99 Dresden Germany, October Proceedings, Coputer Science 1768. pp. 61- 76, 1999.

[Zollner98] Zollner J., H. Federrath, H. Klimant, A. Pfitzmann, R. Piotraschke, A. Westfeld, G. Wicke, G. Wolf, Modelling the Security of Steganographic Systems, Information Hiding, 2nd International Workshop, IH'98 Portland, Oregon, USA, Computer Science 1525. pp. 344-354, April 1998.

\section{(C) 2003 International Journal of Digital Evidence}

\section{ABOUT THE AUTHORS}

Kevin Curran is a Lecturer at the University of Ulster in Northern Ireland. Kevin is a member of the Editorial Committee of The Technology Source, IEEE Computer, Journal of Communications and Networks, IEEE Network Journal (USA), IEEE/ACM Transactions on Networking (USA), Computers and Education and the prestigious Journal of Universal Computer Science (J.UCS). His research interests include distributed computing especially emerging trends within wireless ad-hoc networks, distributed objects, dynamic protocol stacks, multimedia transport protocols and mobile systems. He can be contacted at kj.curran@ulster.ac.uk.

Karen Bailey is a lecturer at the Institute of Technology in Letterkenny, Ireland. She holds a BSc. in Applied Sciences, a master of Philosophy and has just completed a MSc. in Computing and Information Systems. Karen is an active researcher and her research interests include Brewing, Fermentation and Starch Analysis, Steganography, Digital Watermarking, Encryption and Image Processing for Data Security and Data Hiding. Her e-mail is : karen.bailey@,lyit.ie. 